\title{
MBD2 serves as a viable target against pulmonary fibrosis by inhibiting macrophage M2 program
}

\author{
Yi Wang1,2+, Lei Zhang',2+, Guo-Rao Wu',2, Qing Zhou', Huihui Yue ${ }^{1,2}$, Li-Zong Rao, ${ }^{1,}$, Ting Yuan ${ }^{1,3}$,

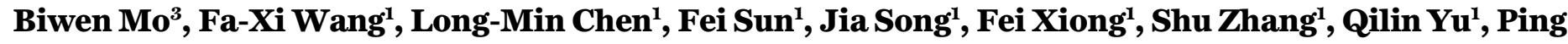

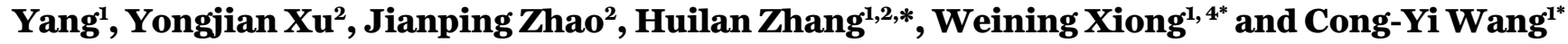 \\ ${ }^{1}$ The Center for Biomedical Research, Tongji Hospital Research Building, Tongji Hospital, Tongji Medical College, Huazhong University of Science and Technology, Wuhan, \\ China. ${ }^{2}$ Department of Respiratory and Critical Care Medicine, Tongji Hospital, Tongji Medical College, Huazhong University of Sciences and Technology, 1095 Jiefang Ave, \\ Wuhan 430030, China. ${ }^{3}$ Department of Respiratory and Critical Care Medicine, the Second Affiliated Hospital of Guilin Medical University, 212 Renmin Road, Guilin 541000 , \\ China. ${ }^{4}$ Department of Respiratory Medicine, Shanghai Ninth People's Hospital, Shanghai Jiaotong University School of Medicine, 639 Zhizaoju Lu, Shanghai, 200011, China. \\ tThese authors contributed equally to this work. \\ *Corresponding author. Email: wangcy@tjh.tjmu.edu.cn, xiongdoctor@qq.com, huilanz_76@163.com
}

Despite past extensive studies, the mechanisms underlying pulmonary fibrosis (PF) still remain poorly understood. Herein we demonstrated that lungs originated from different types of PF patients including coronavirus disease 2019 , systemic sclerosis associated interstitial lung disease and idiopathic pulmonary fibrosis, and mice following bleomycin (BLM)-induced PF are characterized by the altered methyl-CpGbinding domain 2 (MBD2) expression in macrophages. Depletion of Mbd2 in macrophages protected mice against BLM-induced PF. Mbd2 deficiency significantly attenuated transforming growth factor $\beta 1$ (TGF- $\beta 1$ ) production and reduced M2 macrophage accumulation in the lung following BLM induction. Mechanistically, Mbd2 selectively bound to the Ship promoter in macrophages, by which it repressed Ship expression and enhanced $\mathrm{PI}$ KK/Akt signaling to promote macrophage M2 program. Therefore, intratracheal administration of liposomes loaded with $M b d 2$ siRNA protected mice from BLM-induced lung injuries and fibrosis.

Together, our data support that MBD2 could be a viable target against pulmonary fibrosis in clinical settings.

\section{INTRODUCTION}

Pulmonary fibrosis (PF) is a type of chronic, progressive and irreversible lung interstitial disease, which poses a huge threat to public health (1). Some patients develop PF with identifiable triggers such as systemic sclerosis associated interstitial lung disease (SSc-ILD) (2) and 2019 novel coronavirus disease (COVID-19) (3), while some with unknown origins, a term 'idiopathic' is applied (IPF) (2). Despite significant advances over the past few decades, the molecular mechanisms underlying $\mathrm{PF}$ remain poorly understood $(4,5)$, which rendered its treatment largely unsuccessful. Macrophages are crucially important sentinels integral to host pulmonary defense $(6,7)$ characterized by their plasticity and diversity (8). They are either polarized to the classically activated phenotype (M1) (9) or an alternatively activated phenotype (M2) depending on their resided microenvironment (10). Previous studies including ours demonstrated that macrophages, particularly M2 macrophages, contribute to the pathogenesis of pulmonary fibrosis $(6,11)$. Indeed, $\mathrm{M} 2$ macrophages are a major source of TGF- $\beta 1$ and platelet derived growth factor (PDGF), which in turn induce the differentiation of fibroblast into myofibroblast to initiate pulmonary fibrosis (12). Therefore, the number and phenotype of macrophages are thought to be crucial for PF pathological processes $(13,14)$.

There is compelling evidence that DNA methylation, one of the major epigenetic mechanisms, is involved in the pathogenesis of PF $(15,16)$. It has been well recognized that the information encoded by DNA methylation is read by a family of methyl-CpG-binding domain (MBD) proteins (i.e., MBD1, MBD2, MBD3, MBD4, and MeCP2) (17). Each of these proteins, with the exception of MBD3, mediates transcriptional repression or activation by directly binding to the methylated CpG DNA and recruiting proteins to form a suppressive complex (18). Among which, MBD2 has been shown to possess the highest binding activity to the methylated CpG DNA. Therefore, MBD2 has been recognized to be involved in the pathogenesis of tumorigenesis (19), autoimmunity $(20,21)$, ischemic injury (22), obesity (17), and neuronal degeneration (23).

Interestingly, MBD2 was also found to be highly expressed in macrophages in the lungs derived from patients with COVID-19, SSc-ILD or IPF and mice following bleomycin (BLM)-induced pulmonary fibrosis. Moreover, loss of $M b d 2$ in macrophages significantly protected mice from BLMinduced pulmonary fibrosis along with a marked reduction 
of M2 macrophage accumulation in the lung. Mechanistically, Mbd2 selectively bound to the methylated CpG DNA in the SH2-containing inositol 5 '-phosphatase (Ship) promoter, by which it regulated PI3K/Akt signaling to enhance macrophage M2 program. Therefore, intratracheal administration of liposomes carrying $M b d 2$ siRNAs significantly protected mice from BLM-induced pulmonary fibrosis by targeting macrophage M2 program.

\section{RESULTS \\ MBD2 is overexpressed in pulmonary fibrotic lungs with different pathoetiologies}

We first examined MBD2 expression in fibrotic lungs with different origins. Interestingly, MBD2 was almost undetectable in the lung sections originated from normal controls, but it was highly expressed in the lungs originated from SSc-ILD patients (Fig. 1A) and IPF patients (Fig. 1B), while co-immunostaining of CD206 suggested that MBD2 was predominantly co-localized in M2 macrophages, and immunohistochemical staining further confirmed this observation (fig. S1). Next, we checked MBD2 expression in fibrotic lungs derived from severe case of COVID-19 patients (24). As expected, COVID-19 virus could be detected in the lung sections (Fig. 1C), and H\&E and Masson staining indicated the existence of inflammatory infiltration (Fig. 1D) and pulmonary fibrosis (Fig. 1E). Particularly, lungs from COVID-19 patients were also featured by the significant M2 macrophage infiltration (Fig. $1 \mathrm{~F}$ ) along with MBD2 overexpression in M2 macrophages (Fig. 1G). Collectively, these findings suggest that altered MBD2 expression in M2 macrophages could be a common feature in pulmonary fibrosis with different pathological origins.

To address the above assumption, we further examined Mbd2 expression in the lungs of mice following BLM-induced fibrosis. It was noted that BLM time-dependently induced Mbd2 overexpression in the lung. Specifically, an 8-fold Mbd2 overexpression was noted in the fibrotic lungs following day 21 of BLM induction along with increased expression of Collagen I (Col I) and a-SMA, markers for pulmonary fibrosis (Fig. $1 \mathrm{H})$. Moreover, the progression of pulmonary fibrosis was highly correlated with the severity of M2 macrophage accumulation and the levels of Mbd2 overexpression in the lungs (Fig. 1I). Together, our data support that pulmonary fibrosis with different origins are characterized by the induction of MBD2 overexpression in M2 macrophages.

\section{Mbd2 deficiency protects mice against BLM-induced lung injury and fibrosis}

To dissect the role of MBD2 in the pathogenesis of pulmonary fibrosis, we generated a macrophage specific $M b d 2 \mathrm{KO}$ mouse model which selectively deleted $M b d 2$ in macrophages (the

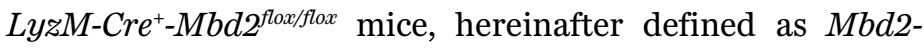

CKO mice), and their littermates (the $L y z M-C r e^{-}-M b d 2^{f l o x} / f l o x$ mice, hereinafter defined as $M b d 2-C$ mice) were served as controls (Fig. 2A). Mbd2 depletion were confirmed by genotyping of the tail blood DNA for the presence of the flox allele (indicated by arrows, fig. S2A) along with the detection of $\mathrm{Cre}$ allele (fig. S2B). Indeed, co-immunostaining of lung sections validated $M b d 2$ deficiency in $\mathrm{F} 4 / 80^{+}$macrophages in $M b d 2-$ CKO mice evidenced by the absence of Mbd2 co-localization with macrophages observed in $M b d 2-C$ mice (Fig. 2B).

The $M b d 2-C K O$ and $M b d 2-C$ mice were subjected to assess the severity of pulmonary fibrosis 21 days following BLM challenge. Significantly attenuated lung injury and fibrosis were noted in $\mathrm{Mbd2-CKO}$ mice as illustrated by the H\&E, Sirius red and Masson's trichrome staining (Fig. 2C, left panel). Phenotypically, the severity of pulmonary fibrosis was much lower in $M b d 2-C K O$ mice as demonstrated by the lower fibrotic scores (Fig. 2C, right panel). In line with these observations, higher severity of fibrosis in $M b d 2-C$ mice was characterized by the higher levels of hydroxyproline than that in $M b d 2-C K O$ mice (Fig. 2D). Consistently, Western blot analysis confirmed a significant reduction for the expression of fibronectin (Fib), Col I and $\alpha$-SMA in the lungs derived from $M b d 2-C K O$ mice (Fig. 2E), and similar data were also obtained by RT-PCR analysis (Fig. 2F). In consistent with published data (25), the LyzM-Cre expression alone did not affect macrophage M2 program and BLM-induced pulmonary fibrosis (fig. S3). Together, our data support that specific loss of $M b d 2$ in macrophages protects mice against BLM-induced lung injury and fibrosis.

\section{Mbd2 deficiency suppresses TGF- $\beta$ signaling}

Given the critical role of TGF- $\beta 1$ played in the initiation and progression of pulmonary fibrosis, we examined TGF- $\beta 1$ expression in the lung. Impressively, $M b d 2-C K O$ mice displayed much lower levels of TGF- $\beta 1$ expression both at protein (Fig. 3A) and mRNA (Fig. 3B) levels than that of lungs from $\mathrm{Mbd2-}$ $C$ mice following BLM induction. These results rendered us to further check the TGF- $\beta 1$ downstream signaling molecules. Indeed, significantly higher levels of phosphorylated Smad2 (p-Smad2) and p-Smad3 were detected in the BLM-induced lungs originated from $M b d 2-C$ mice than that from $M b d 2$ CKO mice, while no perceptible difference in terms of total Smad2/3 was observed (Fig. 3C). Moreover, Tgfbr1 and $T g f b r 2$, the two major TGF- $\beta 1$ receptors, did not show a substantial difference in mRNA levels between lungs from $M b d 2$ $C$ and $M b d 2-C K O$ mice following BLM challenge (Fig. 3, D and E), indicating that the reduced Smad signaling is likely caused by the decreased TGF- $\beta 1$ expression in Mbd2-CKO mice. Remarkably, immunostaining revealed that TGF- $\beta 1$ was predominantly localized in the infiltrated macrophages after BLM induction as determined by F4/80 co-staining (Fig. $3 \mathrm{~F})$. Collectively, our data suggest that $M b d 2$ deficiency 
attenuates the capacity of macrophages for secretion of TGF$\beta 1$, thereby repressing Smad2/3 signaling following BLM induction.

\section{Mbd2 deficiency attenuates the M2 program in macro- phages}

The above results prompted us to embark on the impact of $M b d 2$ deficiency on macrophage M2 program. To confirm the immunostaining results (Fig. 1I), lung $\mathrm{F} 4 / 80^{+}$cells were subjected to flow cytometry analysis as described following BLM induction. Indeed, $\mathrm{Mbd2}$-CKO mice manifested a significantly lower percentage of lung M2 macrophages $\left(\mathrm{F} 4 / 80^{+} \mathrm{CD} 206^{+}\right)$ than that of $M b d 2-C$ mice, but $M b d 2$ deficiency did not seem to affect the induction of M1 macrophages as we failed to detect a perceptible difference in terms of the number of $\mathrm{F} 4 / 80^{+} \mathrm{CD}_{11 \mathrm{C}^{+}}$macrophages (Fig. 4A). Consistently, Western blot analysis of BLM-induced lung homogenates revealed a 2.6-fold reduction for the expression of M2 marker Arg 1 in $M b d 2-C K O$ mice as compared to the $M b d 2-C$ mice (Fig. 4B), and RT-PCR analysis of two additional M2 markers, found in inflammatory zone 1 (Fizz1) (Fig. 4C) and chitinase 3-like 3 (YM1) (Fig. 4D) demonstrated similar results.

To further dissect the mechanism underlying Mbd2 regulation of macrophage M2 program, bone marrow-derived macrophages (BMDMs) were generated from $M b d 2-C$ and $M b d 2-C K O$ mice and then subjected to IL-4 stimulation. Remarkably, IL-4 induced a significantly higher levels of mean fluorescence intensity (MFI) for CD206 in $M b d 2-C$ BMDMs than that in $M b d 2-C K O$ BMDMs (Fig. 4E), and a substantial increase in Mbd2 protein expression was noted upon IL-4 stimulation in $M b d 2-C$ BMDMs, while $\operatorname{Arg} 1$ was decreased in $M b d 2-C K O$ BMDMs as compared to $M b d 2-C$ BMDMs following IL-4 induction (Fig. 4F). RT-PCR analysis of two other M2 markers, Fizz1 (Fig. 4G) and YMI (Fig. 4H) revealed similar results. Next, $M b d 2-C K O$ BMDMs were transduced with mock or Mbd2 lentiviruses followed by IL-4 stimulation. As expected, compared to mock transduced BMDMs, Mbd2 transduced cells were featured by the significantly increased expression of Arg 1 during the course of IL-4 stimulation (fig. S4). Notably, consistent with previous data in the lung, $M b d 2$ deficiency did not affect the induction of M1 macrophages (Fig. 4I) and $I L-6$ expression (Fig. 4J) following LPS and IFN$\checkmark$ stimulation, suggesting that Mbd2 might selectively modulate the M2 program in macrophages.

The above results support the assumption that $M b d 2$ deficiency protects mice against BLM-induced lung injury and fibrosis depending on the repression of macrophage M2 program. To address this question, we first compared disease severity after depletion of macrophages. Clodronate liposomes were intratracheally administered to deplete macrophages in the lungs of $M b d 2-C K O$ and $M b d 2-C$ mice, and mice administered with liposomes were served as controls. Of note, similar as published data (11), macrophages were almost undetectable in the broncho-alveolar lavage fluid (BALF) of clodronate liposome-treated mice along with a significant reduction for the number of total cells (fig. S5). Therefore, one day after depletion of macrophages, the mice were induced for pulmonary fibrosis with BLM, respectively. As expected, $M b d 2-C K O$ mice displayed comparable disease severity as that of $\mathrm{Mbd2-}$ $C$ mice (Fig. 4, $\mathrm{K}$ and L). Importantly, higher disease severity was noted in $M b d 2-C K O$ mice treated with liposomes as compared to that of $M b d 2-C K O$ mice treated with clodronate liposomes (fig. S6). Next, BMDMs from $M b d 2-C$ mice were treated with IL-4 to induce M2 macrophages, and $1 \times 10^{6} \mathrm{M} 2$ macrophages were adoptively transferred through intratracheal injection into clodronate liposomes-treated $M b d 2-C$ and $M b d 2-C K O$ mice on day 7 of BLM induction. Similar as above, adoptive transfer of WT M2 macrophages significantly impaired the protective effect conferred by $M b d 2$ deficiency in $M b d 2-C K O$ mice (Fig. 4, $\mathrm{M}$ and N). Together, these results suggest that $M b d 2$ deficiency protects mice from BLMinduced lung injury and fibrosis dependent on its suppressive effect on macrophage M2 polarization.

\section{Depletion of Mbd2 represses PI3K/Akt signaling to at- tenuate M2 program}

It is believed that PI3K/AKT and STAT6/PPAR- $\gamma$ signaling are critical for the optimal and sustained macrophage M2 program upon IL-4 or IL-13 stimulation $(26,27)$. We then compared temporal expression changes in terms of PI3K/AKT and STAT6/PPAR-Y signaling between $M b d 2-C K O$ and $M b d 2-C$ BMDMs following IL-4 stimulation. No significant difference was observed for total P85 or Akt between $M b d 2-C$ and $M b d 2-C K O$ macrophages. The phosphorylated P85 (p-P85) and p-Akt were relatively low in both types of macrophages before IL-4 stimulation, while a time-dependent increase was observed upon IL-4 stimulation. However, much higher levels of p-P85 and p-Akt (i.e., p-Ser473 and pThr308) were detected in $M b d 2-C$ BMDMs as compared to $M b d 2-C K O$ BMDMs, and IL-4 also time-dependently up-regulated Mbd2 expression (Fig. 5A). Similarly, higher levels of p-P85 and p-AKT were detected in IPF patients as well (fig. S7A). Interestingly, unlike its effect on PI3K/Akt signaling, no significant difference in terms of p-STAT6 and PPAR- $\gamma$ was detected between $M b d 2-C$ and $M b d 2-C K O$ BMDMs following IL-4 stimulation (Fig. 5B).

It has been reported that SHIP, nositolpolyphosphate 4phosphatase type II (Inpp4B) and phosphatase and tensin homolog (Pten) were critical for PI3K/Akt signaling by hydrolyzing or inhibiting the activity of PI-3,4,5-P3 (PIP3) (28, 29). Indeed, $M b d 2-C$ BMDM manifested a 1-fold lower Ship expression than that of Mbd2-CKO BMDMs (Fig. 5C). However, $M b d 2$ deficiency in macrophages did not seem to impact the expression of Inpp4b and Pten (Fig. 5, D and E). 
As MBD2 acts as a reader to interpret DNA methylomeencoded information, we thus next conducted studies to check global DNA methylation levels and Ship promoter methylation state in macrophages. Interestingly, unlike reduced global DNA methylation levels in fibrotic lung genomic DNA, IL-4 induced a global genomic DNA hypermethylation in BMDMs (Fig. 5F). Particularly, IL-4 induced the Ship promoter to undergo a DNA hypermethylation (Fig. 5G) and similar results were noted in IPF patients as well (fig. S7B). The next key question is whether Mbd2 selectively binds to the above hypermethylated CpG DNA within the Ship promoter. Chromatin immunoprecipitation (ChIP) was then employed to address this question. Indeed, Mbd2 selectively bound to the methylated CpG DNA in 5 regions (fig. S8, from $-1336 \mathrm{bp}$ to $+167 \mathrm{bp}$, the transcriptional start site as +1 ) within the Ship promoter, while CHIP-PCR yielded negative result in the distal region absent of CpG DNA (Fig. 5H). Furthermore, DNA methylation dependent luciferase reporter assays confirmed that the transcriptional activity of Ship was higher in the BMDMs with mutant plasmid (no methylated DNA) than those with the WT plasmid (with methylated DNA) (Fig. 5I). Importantly, suppression of Ship by siRNA in $M b d 2-C K O$ BMDMs restored their capability for M2 polarization following IL-4 stimulation (Fig. 5J). Collectively, our data support that MBD2 binds to the Ship promoter to repress its expression, by which it enhances PI3K/Akt signaling to promote macrophage M2 program.

\section{Intratracheal administration of Mbd2 siRNA lipo- somes protects mice against BLM-induced pulmonary fibrosis}

Finally, we sought to translate the above discoveries into a therapeutic approach against pulmonary fibrosis. To this end, we generated lipid-based liposomes loaded with an $M b d 2$ siRNA (fig. S9A). The prepared liposomes displayed $>90 \%$ entrapment efficiency for loading siRNA with a Zetapotential of $3.2 \mathrm{mv}$ (fig. S9B). A representative image was then taken by transmission electron microscope (TEM) (fig. S9C), and those liposomes manifested a normal distribution of hydrodynamic diameter (fig. S9D) with sustained stability within 24 hours (fig. S9E). Next, CCK-8 assay was used to check the effect of liposome-loaded siRNA on cell viability of BMDMs, and we failed to detect any toxicity of liposomeloaded siRNA in cell viability with different concentrations (fig. S9F). To assess the safety of liposome in vivo, we conducted HE staining and TUNEL assay of lung sections following intratracheal liposome administration. As expected, administration of liposomes did not induce inflammation in the lung and epithelial apoptosis (fig. S9, G and H). To demonstrate the biodistribution of liposomes, IVIS ${ }^{\circledast}$ spectrum imaging system was employed to track the fluorescent signal of DiR-labeled liposomes. Encouragingly, those intratracheal administered liposomes sustainably accumulated in the lung for at least 7 days (Fig. 6A). To further confirm this observation, organs such as heart, liver, spleen, lung and kidney were collected from 4 mice after day 7 of intratracheal injection of liposomes, and then subjected to analysis of near infrared fluorescent (NIRF) signals. As expected, NIRF signals were only detected in the lungs, but absent in other organs (Fig. 6B).

Next, the distribution of liposomes in the lung tissues was further explored in a BLM-induced mouse model. Surprisingly, liposomes were predominantly located in the fibrotic area of lung tissues and could be mainly overlapped with $\mathrm{F} 4 / 80^{+}$macrophages but not prosurfactant protein $\mathrm{C}$ (pro$\mathrm{SPC})^{+}$epithelial cells, indicating a high efficiency for their uptake by macrophages (Fig. 6C, fig. S10). To further address the effect of siRNA-loaded liposomes on protein expression in lung tissues, we examined Mbd2 expression changes in the lungs following liposome-based pulmonary delivery. Of note, a significant decrease in Mbd2 expression was noted after intratracheal delivery of $M b d 2$ siRNA-loaded liposomes, and the nadir was detected at day 3 after the treatment. Nevertheless, this expression was returned to normal levels at day 7 (Fig. 6D).

Finally, the therapeutic effect of siRNA-loaded liposomes was assessed in mice following BLM induction. WT mice were administrated with Scrambled or Mbd2 siRNA-loaded liposomes at day 14 and day 18 ( $1 \mathrm{mg} / \mathrm{kg}$; i.t.), respectively (Fig. $6 \mathrm{E})$. Indeed, administration of $M b d 2$ siRNA-loaded liposomes significantly alleviated BLM-induced lung injury and fibrosis as illustrated by the histopathological analysis (Fig. 6F, left panel) and fibrotic scores (Fig. 6F, right panel). Consistently, mice administered with $M b d 2$ siRNA-loaded liposomes displayed much lower levels of hydroxyproline in the lung (Fig. $6 \mathrm{G})$, coupled with a significant reduction for the expression of fibrotic markers (fibronectin, collagen I and $\alpha$-SMA) and macrophage M2 marker, Arg 1 (Fig. $6 \mathrm{H}$ ). Together, our data support that intratracheal administration of $M b d 2$ siRNAloaded liposomes could be a viable therapy against pulmonary fibrosis in clinical settings.

\section{DISCUSSION}

In the present report, we conducted studies in patients and animals to address the impact of MBD2, a reader for interpreting DNA methylation encoded information, on the pathogenesis of pulmonary fibrosis. Lungs originated from different types of PF patients and mice following BLMinduced PF were characterized by the altered MBD2 expression. Specifically, MBD2 was overexpressed in infiltrated M2 macrophages within the lung during the course of fibrotic processes. Therefore, depletion of $M b d 2$ in macrophages protected mice from BLM-induced lung injury and pulmonary fibrosis. In general, macrophages are characterized by the 
DNA hypermethylation in the fibrotic lungs. As a result, Mbd2 binds to the methylated CpG DNA within the Ship promoter to inhibit Ship expression, by which Mbd2 enhances PI3K/Akt signaling to promote macrophage M2 program. M2 macrophages in turn secrete large amount of TGF- $\beta 1$, which then activates fibroblasts to initiate and promote fibrotic processes (Fig. 6I). Indeed, mice with macrophage specific $M b d 2$ deficiency are characterized by the reduced TGF- $\beta 1$ production along with attenuated Smad2/3 signaling following BLM challenge. Together, our results support that strategies aimed at silencing MBD2 in macrophages could be a viable therapy against pulmonary fibrosis in clinical settings.

In general, $\mathrm{PF}$ is featured by idiopathic in nature with possible genetic predisposition. However, it can also be recognized as a complication of connective tissue disease or with certain environmental, occupational or drug exposures. For example, $\mathrm{PF}$ is a common consequence in certain patients following COVID-19 infection $(30,31)$. Unfortunately, no effective pharmacological therapy against PF thus far is available other than lung transplantation. In this report, we demonstrated evidence that macrophages originated from fibrotic lungs of patients with COVID-19 and SSc-ILD or IPF, are featured by the overexpression of MBD2, a methylated DNA binding protein. In fact, those observations are consistent with previous studies (32), in which PF patients manifest a DNA methylation turnover characterized by the changes of methylation patterns for the target genes and/or methylation levels for a particular gene. For example, the levels of DNA methylation enzymes such as DNMT3a and DNMT3b, and MBD proteins such as MeCP2, are highly elevated in the lungs of IPF patients, which were associated with up-regulation of fibrotic genes $(15,33)$. Collectively, these data support that DNA methylation is implicated in PF pathogenesis, and MBD2 serves as a reader of methylated DNA contributing to PF development.

The most exciting discovery in this report is that $\mathrm{Mbd} 2$ was noted to regulate macrophage M2 program, which maybe a common factor for the initiation of fibrotic processes. Previous studies suggested that DNA methylation may modulate the activation of M2 macrophages during the course of obesity (34). We now provided convincing evidence that loss of MBD2 promotes macrophage M2 polarization. Specifically, we demonstrated that mice with specific deficiency of $M b d 2$ in macrophages were significantly protected from BLM-induced lung injury and fibrosis, which were abolished once macrophages were depleted or $M b d 2-C$ M2 macrophages were adoptively transferred into the lung. Moreover, MBD2 only selectively regulates macrophage M2 program, without a perceptible impact on M1 macrophages. In addition, we also observed that loss of $M b d 2$ in macrophages did not impact the total number of macrophages in the lung (fig. S11). It was noted that IL-4 stimulates the Ship promoter in macrophages to undergo a DNA hypermethylation along with MBD2 overexpression, and MBD2 in turn binds to the methylated CpG DNA within the Ship promoter to suppress its expression. DNA methylation dependent promoter reporter assays indeed confirmed that MBD2 significantly suppressed Ship transcription. The attenuated Ship expression would then release its suppressive effect on the PI3K/Akt signaling (35), which is essential to activate and sustain macrophage M2 program (36). Of importantly note, our macrophage depletion and adoptive transfer studies further confirmed that the reduced M2 macrophages contribute to the protective effect on pulmonary fibrosis conferred by $M b d 2$ deficiency. As a result, mice deficient in $M b d 2$ are manifested by the significant reduction of M2 macrophages in the lung following BLM induction.

Given the fact that no effective therapy is currently available for PF, numerous clinical trials have been carried out to characterize viable drugs $(37,38)$. For this purpose, properties for a particular drug such as half-life in the blood, concentrations in the lungs, and possible side effects on other organs and tissues, are necessary for consideration. Therefore, drug administration directly through tracheal inhalation rather than systemic delivery could be a better approach for PF patients. Liposomes are established drug carriers for inhalation owing to their safety and ability to provide controlled drug release in the lung. These carriers can entrap a wide range of therapeutic molecules for delivery in large volumes to the peripheral airways using medical nebulizers (39, 40). Indeed, a unique delivery system with nanostructured lipid carriers (NLC) through inhalation of prostaglandin E2 (PGE2) coupled with MMP3 siRNAs, CCL12 (chemokine), and HIF1A, has been employed to limit lung injuries in PF patients, and to prevent disease progression by suppressing the synthesis of pro-fibrotic proteins $(41,42)$. Similarly, our studies in this report demonstrated that liposomes carrying the Mbd2 siRNA could be efficiently uptaken by the macrophages in fibrotic lesions of the lung following intratracheal injection. More excitingly, the liposome-based repression of Mbd2 expression lasted for a week or so. As a result, intratracheal delivery of liposomes carrying the $M b d 2$ siRNA protected mice from BLM-induced lung injury and fibrosis.

Interestingly, immunostaining of lung sections from BLM-induced fibrotic mice also noted MBD2 overexpression in certain cells other than $\mathrm{F} 4 / 80^{+}$macrophages (Fig. 3F), while analysis of IPF single cell sequencing data suggested that those cells were fibroblasts and epithelial cells (fig. S12). Given that this report selectively focused on macrophages, the impact of altered MBD2 expression in fibroblasts and epithelial cells on pulmonary fibrosis would be tackled in our future studies.

In summary, we have demonstrated evidence that lungs originated from PF patients and mice following BLM-induced 
PF are characterized by the altered MBD2 expression. Therefore, mice with macrophages deficient in $M b d 2$ are protected from BLM-induced lung injury and fibrosis. MBD2 selectively binds to the methylated CpG DNA within the Ship promoter in macrophages, by which it represses Ship expression and enhances PI3K/Akt signaling to promote macrophage M2 program. Since MBD2 itself does not affect DNA methylation and appears to be dispensable for biological processes under physiological condition (17, 20,22), MBD2 could be, therefore, a viable target against PF. Indeed, intratracheal administration of liposomes carrying the $M b d 2$ siRNA substantially protected mice from BLM-induced lung injuries and fibrosis. Together, our data support that intratracheal administration of liposome-based MBD2 siRNA could be a viable therapy against pulmonary fibrosis in clinical settings.

\section{Materials and Methods \\ Human samples}

Lung tissues from non-small cell lung cancer (NSCLC, $\mathrm{n}=6$ ), COVID-19 patients $(n=2)$, SSc-ILD patients $(n=3)$ and IPF patients $(\mathrm{n}=8)$ were collected in Tongji hospital with informed consent. COVID-19 was diagnosed by nucleic acid test (43), SSc-ILD classified according to ACR/EULAR criteria (44) and the IPF diagnosis was made according to the ATS/ERS consensus diagnostic criteria (45). The studies were approved by the Human Assurance Committee of Tongji Hospital. Clinical data and pulmonary function tests were provided in Table S1.

\section{Bleomycin (BLM) induction of pulmonaryfibrosis}

$M b d 2^{f l o x / f l o x}$ mice were generated using the CRISPR-Cas9 system by the Bioray Laboratories Inc (Shanghai, China). Two loxP sequences were inserted in the introns flanked with the exon 2 of MBD2 as described in Fig. 2A. The LyzM-Cre transgenic mice were purchased from the Jackson's Laboratory (Bar Harbor, ME, USA) and bred into C57BL/6 background by backcrossing with wild-type (WT) C57BL/ 6 mice for more than 12 generations. $L y z M-C r e^{+}-M b d 2^{f l o x} f f l o x(M b d 2-C K O)$ mice were generated by crossing the LyzM-Cre mice with $M b d 2^{f l o x f f l o x}$ mice for specific deletion of Mbd2 in macro-

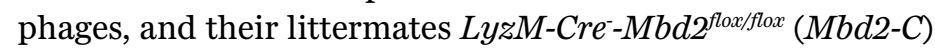
were used as controls. WT C57BL/6 mice were derived from the Jackson's Laboratory. $M b d 2-C$ and $M b d 2-C K O$ mice (8-10 weeks old) were anesthetized with $1 \%$ pentobarbital sodium $(60 \mathrm{mg} / \mathrm{kg})$ and then intratracheally administered $0.5 \mathrm{mg} / \mathrm{kg}$ BLM (Nippon Kayaku, Tokyo, Japan) in 40ul of normal saline (11). Mice administered with same volume of normal saline served as controls. siRNA-loaded liposomes were injected into the anesthetized animals via intratracheal on the days 14 and 18 after BLM injection. The mice were sacrificed on day 21 following BLM challenge to analyze pulmonary fibrosis. All mice were housed in an SPF facility at the Tongji Medical
College with a 12/12h light/dark cycle. All experimental procedures were approved by the Animal Care and Use Committee at the Tongji Hospital.

Fluorescent images were acquired on an IVIS Lumina XR system (Caliper Life Sciences, Hopkinton, USA) equipped with a 150-W quartz halogen lamp and a 1-mW power scanning laser. The following parameters were used: exposure time $0.5 \mathrm{~s}$, binning $1, \mathrm{f} / \mathrm{stop} 2$. Filter sets were fixed with the following parameters: excitation wavelength at $640 \mathrm{~nm}$ and emission wavelength at $670 \mathrm{~nm}$. Acquired images were measured and analyzed by the Living Imaging ${ }^{\circledR}$ software.

\section{Reagents and antibodies}

Murine recombinant IL-4 was obtained from PeproTech (London, United Kingdom). Antibodies against Collagen type I was purchased from EMD Millipore (Schwalbach, Germany). Antibodies against Fibronectin, $\alpha$-SMA, Arginase-1, CD68, MBD2 and Prosurfactant Protein C (pro-SPC) were purchased from Abcam (Cambridge, MA, USA), and antibodies against p-Smad2, p-Smad3, STAT6, P85, p-P85 and p-Akt (Thr308) were obtained from Cell Signaling (Danvers, MA, USA). Antibodies against MBD2, GAPDH, p-Akt (Ser473) and p-STAT6 were obtained from Santa Cruz Biotechnology (Santa Cruz, CA, USA). Anti-mouse F4/80-PerCP/Cy5.5 and CD206-FITC were from Biolegend (San Diego, CA, USA), and CD11c-APC were purchased from BD Biosciences (San Jose, CA, USA). Lipidoid (C12-200) was purchased from Xinjiahecheng Medical Chemistry Corporation (Wuhan, Hubei, China). mPEG2000-DEG was purchased from NOF Corporation (Tokyo, Japan). All other reagents were purchased from Sigma (St Louis, MO, USA), unless otherwise stated.

\section{Histological and immunohistochemical analysis}

The left lung was inflated and placed in fresh $4 \%$ neutral buffered paraformaldehyde for $24 \mathrm{~h}$ at room temperature, embedded in paraffin and subjected to the histological analysis as previously reported (17). Each successive field was individually assessed for the severity of interstitial fibrosis in a blinded fashion by two pathologists using the Ashcroft scoring system (46). For immunostaining, the frozen sections $(7 \mu \mathrm{m})$ were probed with antibodies against CD206, CD68, $\mathrm{Mbd} 2$ and F4/80, followed by staining with Alexa Fluor 594labeled anti-mouse/rabbit or Alexa Fluor 488-conjugated anti-rabbit/mouse antibodies (Invitrogen, Carlsbad, CA, USA), respectively. COVID-19 viral immunostaining was conducted as previously reported (47).

\section{Culture and treatment of bone marrow-derived macro- phages (BMDMs)}

BMDMs were obtained from male mice as reported $(48,49)$. Red blood cells in bone marrow samples were lysed, and then resuspended in $50 \mathrm{ml}$ of culture medium consisting of RPMI 
1640, 10\% fetal bovine serum (FBS), penicillin/streptomycin, and $30 \mathrm{ng} / \mathrm{ml}$ macrophage colony-stimulating factor (M-CSF). The cells were next plated in $35 \times 15 \mathrm{~mm}$ tissue culture dishes at $37^{\circ} \mathrm{C}$, and the medium was changed every 2 days. After 7 days, the differentiated macrophages were co-cultured with IL-4 $(10 \mathrm{ng} / \mathrm{ml})$ at the indicated time points.

\section{Macrophage depletion and adoptive transfer studies}

Clodronate liposomes $(40 \mu \mathrm{l})$ were intratracheally administered for two successive days before BLM induction, and the severity of pulmonary fibrosis was assessed 21 days after BLM induction (11). For adoptive transfer studies, WT naive BMDMs were first stimulated with IL-4 (10ng/ml) for 12 hours to induce M2 macrophages, and then transferred into the lungs of clodronate liposome-treated $M b d 2-C$ and $M b d 2-$ CKO mice at a density of $1 \times 10^{6}$ cells/mouse (50 $\left.\mathrm{ll}\right)$ at day 7 of BLM induction through intratracheal injection. The mice were sacrificed 2 weeks after the adoptive transfer for fibrotic analysis.

\section{Western blot analysis}

Lung tissues and cultured cells were homogenized in RIPA lysis buffer (Beyotime, Shanghai, China), and equal amounts of lysates were separated on $10 \%$ polyacrylamide gels (Sigma) and transferred onto PVDF membranes. The membranes were next probed with indicated primary antibodies for analysis of protein levels as described (50).

\section{Quantitative RT-PCR analysis}

Quantitative RT-PCR was performed using the SYBR Premix Ex Taq (TaKaRa, Tokyo, Japan), and the relative expression of each target gene was normalized by $\beta$-actin as previously described $(51,52)$. The following primers were used for each target gene: Fibronectin (5'-ATG CAA CGA TCA GGA CAC AA -3 ' and $5{ }^{\prime}$-TGT GCC TCT CAC ACT TCC AC $-3{ }^{\prime}$ ); Collagen $I$ ( $5^{\prime}$-CCT GGT AAA GAT GGT GCC- 3 ' and 5 ' -CAC CAG GTT CAC CTT TCG CAC C $\left.-3^{\prime}\right)$; $\alpha-S M A$ (5'-CGT ACA ACT GGT ATT GTG CTG GAC $-3^{\prime}$ and $5^{\prime}$-TGA TGT CAC GGA CAA TCT CAC GCT -3' ); YM1 (5' -GGG CAT ACC TTT ATC CTG AG-3 ' and 5 ' -CCA CTG AAG TCA TCC ATG TC-3' ); Fizz1 (5' -TCC CAG TGA ATA CTG ATG AGA- ${ }^{\prime}$ ' and $5^{\prime}$-CCA CTC TGG ATC TCC CAA GA-3' ); Ship (5' -GAG ACA CTG TTT CAG CGT CTA C-3' and 5'-CGT CTT CAA AAA GTC GGA ATC CA-3'); Inpp $4 B$ (5' -TCC AGA AGA TTC CGA ACG AGC-3' and 5' GTT TCC GAT CAC TGA CAG GAG-3' ); Pten (5' -TGG ATT CGA CTT AGA CTT GAC CT-3' and $5^{\prime}$-GCG GTG TCA TAA TGT CTC TCA G-3'); Mbd2 (5'-GGA GGA AGT GAT CCG AAA ATC AG-3' and $5^{\prime}$-AGC ATT TCC CAG GTA TCT TGC$3^{\prime}$ ); and $\beta$-actin (5' -TGA CGT TGA CAT CCG TAA AGA CC$3^{\prime}$ and 5 '-CTC AGG AGG AGC AAT GAT CTT GA-3' ). Relative expression levels for each target gene were calculated using the $2^{-\Delta \Delta C t}$ method.
Chromatin immunoprecipitation (ChIP) assay and Ship promoter reporter assay

ChIP assays were conducted using a ChIP assay kit (Beyotime, Shanghai, China) as reported (8). Briefly, $1 \times 10^{6}$ WT BMDMs were cross-linked with formaldehyde, and chromatin fragmentation was carried out according to the protocol provided. The above prepared diluted soluble chromatin solution was then incubated with an $\mathrm{Mbd} 2$ antibody overnight at $4^{\circ} \mathrm{C}$ with rotation. Normal rabbit IgG was used to determine nonspecific bindings. The above mixtures were next incubated with protein $\mathrm{A}+\mathrm{G}$ agarose beads, and the protein-DNA complexes were eluted out after washes. The eluted DNA was subjected to ChIP-PCR with indicated primers. The primers used for Ship in the ChIP assay were: F1:5' - TCA GAA TGT AGA CGT GAG CTC TTG T -3', R1: 5 ' - GCT GTG TTG ATG TCA TCC ATG G-3 ' ; F2: 5 ' - TCC CAT GTC CTC CAC ACA CCA G$3^{\prime}$, R2: $5^{\prime}$ - CAC TCC TTC ATC TGA ACC TTG TCA C-3' ; F3: $5^{\prime}$ - AAC AAT CAC CAC TTC TGC CGT AAG C-3 ', R3: $5^{\prime}$ - CCT CTG TGG CTA GAC ATG GCT AT-3' ; F4: $5^{\prime}$ - GGA AGG TAG ATG ATG CCC CC-3' , R4: $5^{\prime}$ - CAG CAA AGT AGT TCA GGG CC-3 ' ; F5: $5^{\prime}$ - GAG TGT CCG TCC TGG GAG TGG-3 ' , R5: 5 ' AGT TGG GAG GAG ACG GGT ACT CAC A-3' . The distal region of the Ship promoter absent of CpG DNA was employed as a negative control (The primer was F: $5^{\prime}$-CAT GAG ATC CAA CTG TAA GGC-3' , R: $5^{\prime}$-CAT ACT GCT GTT CAT CAC CAA-3' ). The dual luciferase reporter system (Promega, Madison, WI) was used for the Ship promoter luciferase reporter assays, in which the Mbd2 binding site within the Ship promoter was disrupted using the established techniques (17).

\section{Global DNA methylation assay and bisulfite DNA se- quencing}

Global DNA methylation was determined using a MethylFlashTM Methylated DNA Quantification Kit (Epigentek, NY, USA) as instructed. Bisulfite DNA sequencing was conducted as previously described (20).

\section{siRNA transfection}

Mbd2-CKO BMDMs cultured in RIPM1640 supplemented with $10 \%$ FBS were transfected with a Ship siRNA or Scrambled siRNA (Ribobio, Guangzhou, China) using the Lipofectamine 3000 reagent (Invitrogen, Carlsbad, CA, USA) as previously described (53). The transfected cells were next stimulated with murine IL-4 $(10 \mathrm{ng} / \mathrm{ml})$ at indicated time points.

\section{Flow cytometry analysis}

Mononuclear cells were isolated from lung tissues using the established techniques (54), while BMDMs were stimulated with LPS $(100 \mathrm{ng} / \mathrm{ml})+\mathrm{IFN}-\mathrm{\gamma}(100 \mathrm{U} / \mathrm{ml})$ or IL-4 $(10 \mathrm{ng} / \mathrm{ml})$ for 12 hours. The cells were then stained with anti-mouse F4/80PerCP/Cy5.5 (1:100), CD11c-APC (1:100) and CD206-FITC 
(1:100) antibodies. After washes, the cells were analyzed using a FACSCanto II (BD Biosciences, San Jose, CA, USA). All data were analyzed using the FACS Express V3 software (De Novo Software) according to the manufacturer's instructions.

\section{Preparation and characterization of siRNA-loaded lip- osomes}

siRNA-loaded liposomes were prepared as reported (55). Briefly, lipidoid, cholesterol, DSPC and MPEG-DMG were dissolved in ethanol at a molar ratio of 50:38.5:10:1.5. siRNA was dissolved in citrated buffer $(10 \mathrm{mM}, \mathrm{pH}=3)$. The lipid components and the dissolved siRNA were then mixed rapidly by vortex. The next step was to exclude unentrapped siRNA by ultrafiltration centrifugation. Finally, the siRNA-liposomes were diluted in PBS. Hydrodynamic diameter, poly dispersity, zeta potential and stability of the liposomes were measured by dynamic light scattering (DLS) (Malvern Zetasizer NanoZS, UK). A Ribogreen assay was employed to calculate the entrapment efficiency of siRNA. After staining with $2 \%$ phosphotungstic acid, the liposomes were characterized by transmission electron microscope (TEM, JEM-1230, Jeol, Japan).

\section{Statistical analysis}

Comparisons between groups were undertaken using the Graph Pad Prism (version 5.0) software (GraphPad Software Inc., San Diego, CA,USA). Two experimental groups were compared using a Student's $t$ test for paired data or a Student's $t$ test with Welch's correction for unpaired data. For comparisons more than two groups, a one-way ANOVA with Bonferroni's correction was used. The data are presented as the mean \pm SD unless otherwise specified. In all cases, $p<$ 0.05 was considered with statistical significance.

\section{REFERENCES AND NOTES}

1. M. J. Schafer, T. A. White, K. lijima, A. J. Haak, G. Ligresti, E. J. Atkinson, A. L. Oberg, J. Birch, H. Salmonowicz, Y. Zhu, D. L. Mazula, R. W. Brooks, H. FuhrmannStroissnigg, T. Pirtskhalava, Y. S. Prakash, T. Tchkonia, P. D. Robbins, M. C. Aubry, J. F. Passos, J. L. Kirkland, D. J. Tschumperlin, H. Kita, N. K. LeBrasseur, Cellular senescence mediates fibrotic pulmonary disease. Nat. Commun. 8, 14532 (2017). doi:10.1038/ncomms14532 Medline

2. A. McLean-Tooke, I. Moore, F. Lake, Idiopathic and immune-related pulmonary fibrosis: Diagnostic and therapeutic challenges. Clin. Transl. Immunology 8, e1086 (2019). doi:10.1002/cti2.1086 Medline

3. Y. H. Xu, J. H. Dong, W. M. An, X. Y. Lv, X. P. Yin, J. Z. Zhang, L. Dong, X. Ma, H. J. Zhang, B. L. Gao, Clinical and computed tomographic imaging features of novel coronavirus pneumonia caused by SARS-CoV-2. J. Infect. 80, 394-400 (2020). doi:10.1016/i.jinf.2020.02.017 Medline

4. G. Raghu, J. Behr, K. K. Brown, J. J. Egan, S. M. Kawut, K. R. Flaherty, F. J. Martinez, S. D. Nathan, A. U. Wells, H. R. Collard, U. Costabel, L. Richeldi, J. de Andrade, N. Khali,, L. D. Morrison, D. J. Lederer, L. Shao, X. Li, P. S. Pedersen, A. B. Montgomery, J. W. Chien, T. G. O'Riordan; ARTEMIS-IPF Investigators*, Treatment of idiopathic pulmonary fibrosis with ambrisentan: A parallel, randomized trial. Ann. Intern. Med. 158, 641-649 (2013). doi:10.7326/00034819-158-9-201305070-00003 Medline

5. L. Shulgina, A. P. Cahn, E. R. Chilvers, H. Parfrey, A. B. Clark, E. C. Wilson, O. P. Twentyman, A. G. Davison, J. J. Curtin, M. B. Crawford, A. M. Wilson, Treating idiopathic pulmonary fibrosis with the addition of co-trimoxazole: A randomised controlled trial. Thorax 68, 155-162 (2013). doi:10.1136/thoraxinl-2012-202403 Medline

6. J. L. Larson-Casey, J. S. Deshane, A. J. Ryan, V. J. Thannickal, A. B. Carter, Macrophage Akt1 Kinase-Mediated Mitophagy Modulates Apoptosis Resistance and Pulmonary Fibrosis. Immunity 44, 582-596 (2016) doi:10.1016/i.immuni.2016.01.001 Medline

7. J. Mesureur, J. R. Feliciano, N. Wagner, M. C. Gomes, L. Zhang, M. Blanco-Gonzalez, M. van der Vaart, D. O'Callaghan, A. H. Meijer, A. C. Vergunst, Macrophages, but not neutrophils, are critical for proliferation of Burkholderia cenocepacia and ensuing host-damaging inflammation. PLOS Pathog. 13, e1006437 (2017). doi:10.1371/journal.ppat 1006437 Medline

8. Y. Wang, J. Zhu, L. Zhang, Z. Zhang, L. He, Y. Mou, Y. Deng, Y. Cao, P. Yang, Y. Su, J. Zhao, S. Zhang, Q. Yu, J. Hu, Z. Chen, Q. Ning, X. Xiang, Y. Xu, C. Y. Wang, W. Xiong, Role of $\mathrm{C} / \mathrm{EBP}$ homologous protein and endoplasmic reticulum stress in asthma exacerbation by regulating the $\mathrm{IL}-4 /$ signal transducer and activator of transcription 6/transcription factor EC/IL-4 receptor $\alpha$ positive feedback loop in M2 macrophages. J. Allergy Clin. Immunol. 140, 1550-1561.e8 (2017). doi:10.1016/j.jaci.2017.01.024 Medline

9. T. Kimura, S. Nada, N. Takegahara, T. Okuno, S. Nojima, S. Kang, D. Ito, K. Morimoto, T. Hosokawa, Y. Hayama, Y. Mitsui, N. Sakurai, H. Sarashina-Kida, M. Nishide, Y Maeda, H. Takamatsu, D. Okuzaki, M. Yamada, M. Okada, A. Kumanogoh, Polarization of M2 macrophages requires Lamtor1 that integrates cytokine and amino-acid signals. Nat. Commun. 7, 13130 (2016). doi:10.1038/ncomms13130 Medline

10. A. J. Byrne, T. M. Maher, C. M. Lloyd, Pulmonary Macrophages: A New Therapeutic Pathway in Fibrosing Lung Disease? Trends Mol. Med. 22, 303-316 (2016). doi:10.1016/j.molmed.2016.02.004 Medline

11. Y. Yao, Y. Wang, Z. Zhang, L. He, J. Zhu, M. Zhang, X. He, Z. Cheng, Q. Ao, Y. Cao, P. Yang, Y. Su, J. Zhao, S. Zhang, Q. Yu, Q. Ning, X. Xiang, W. Xiong, C. Y. Wang, Y. Xu, Chop Deficiency Protects Mice Against Bleomycin-induced Pulmonary Fibrosis by Attenuating M2 Macrophage Production. Mol. Ther. 24, 915-925 (2016). doi:10.1038/mt.2016.36 Medline

12. S. Su, Q. Zhao, C. He, D. Huang, J. Liu, F. Chen, J. Chen, J. Y. Liao, X. Cui, Y. Zeng, H. Yao, F. Su, Q. Liu, S. Jiang, E. Song, miR-142-5p and miR-130a-3p are regulated by IL-4 and IL-13 and control profibrogenic macrophage program. Nat. Commun. 6, 8523 (2015). doi:10.1038/ncomms9523 Medline

13. A. R. Froese, C. Shimbori, P. S. Bellaye, M. Inman, S. Obex, S. Fatima, G. Jenkins, J. Gauldie, K. Ask, M. Kolb, Stretch-induced Activation of Transforming Growth Factor- $\beta 1$ in Pulmonary Fibrosis. Am. J. Respir. Crit. Care Med. 194, 84-96 (2016). doi:10.1164/rccm.201508-16380C Medline

14. D. V. Pechkovsky, A. Prasse, F. Kollert, K. M. Engel, J. Dentler, W. Luttmann, K. Friedrich, J. Müller-Quernheim, G. Zissel, Alternatively activated alveolar macrophages in pulmonary fibrosis-mediator production and intracellular signal transduction. Clin. Immunol. 137, 89-101 (2010). doi:10.1016/j.clim.2010.06.017 Medline

15. Y. Y. Sanders, N. Ambalavanan, B. Halloran, X. Zhang, H. Liu, D. K. Crossman, M. Bray, K. Zhang, V. J. Thannickal, J. S. Hagood, Altered DNA methylation profile in idiopathic pulmonary fibrosis. Am. J. Respir. Crit. Care Med. 186, 525-535 (2012). doi:10.1164/rccm.201201-00770C Medline

16. I. V. Yang, B. S. Pedersen, E. Rabinovich, C. E. Hennessy, E. J. Davidson, E. Murphy, B. J. Guardela, J. R. Tedrow, Y. Zhang, M. K. Singh, M. Correll, M. I. Schwarz, M. Geraci, F. C. Sciurba, J. Quackenbush, A. Spira, N. Kaminski, D. A. Schwartz, Relationship of DNA methylation and gene expression in idiopathic pulmonary fibrosis. Am. J. Respir. Crit. Care Med. 190, 1263-1272 (2014). doi:10.1164/rccm.201408-14520C Medline

17. J. Cheng, J. Song, X. He, M. Zhang, S. Hu, S. Zhang, Q. Yu, P. Yang, F. Xiong, D. W. Wang, J. Zhou, Q. Ning, Z. Chen, D. L. Eizirik, Z. Zhou, C. Zhao, C. Y. Wang, Loss of Mbd2 Protects Mice Against High-Fat Diet-Induced Obesity and Insulin Resistance by Regulating the Homeostasis of Energy Storage and Expenditure. Diabetes 65 3384-3395 (2016). doi:10.2337/db16-0151 Medline

18. Q. Du, P. L. Luu, C. Stirzaker, S. J. Clark, Methyl-CpG-binding domain proteins: Readers of the epigenome. Epigenomics 7, 1051-1073 (2015). doi:10.2217/epi.15.39 Medline

19. M. Zhou, K. Zhou, L. Cheng, X. Chen, J. Wang, X. M. Wang, Y. Zhang, Q. Yu, S. Zhang, 
D. Wang, L. Huang, M. Huang, D. Ma, T. Cheng, C. Y. Wang, W. Yuan, J. Zhou, MBD2 Ablation Impairs Lymphopoiesis and Impedes Progression and Maintenance of TALL. Cancer Res. 78, 1632-1642 (2018). doi:10.1158/0008-5472.CAN-17-1434 Medline

20. J. Zhong, Q. Yu, P. Yang, X. Rao, L. He, J. Fang, Y. Tu, Z. Zhang, Q. Lai, S. Zhang, M. Kuczma, P. Kraj, J. F. Xu, F. Gong, J. Zhou, L. Wen, D. L. Eizirik, J. Du, W. Wang, C. Y. Wang, MBD2 regulates TH17 differentiation and experimental autoimmune encephalomyelitis by controlling the homeostasis of T-bet/HIx axis. J. Autoimmun. 53, 95-104 (2014). doi:10.1016/j.jaut.2014.05.006 Medline

21. P. C. Cook, H. Owen, A. M. Deaton, J. G. Borger, S. L. Brown, T. Clouaire, G. R. Jones, L. H. Jones, R. J. Lundie, A. K. Marley, V. L. Morrison, A. T. Phythian-Adams, E. Wachter, L. M. Webb, T. E. Sutherland, G. D. Thomas, J. R. Grainger, J. Selfridge, A. N. McKenzie, J. E. Allen, S. C. Fagerholm, R. M. Maizels, A. C. Ivens, A. Bird, A. S. MacDonald, A dominant role for the methyl-CpG-binding protein Mbd2 in controlling Th2 induction by dendritic cells. Nat. Commun. 6, 6920 (2015). doi:10.1038/ncomms7920 Medline

22. X. Rao, J. Zhong, S. Zhang, Y. Zhang, Q. Yu, P. Yang, M. H. Wang, D. J. Fulton, H. Shi, Z. Dong, D. Wang, C. Y. Wang, Loss of methyl-CpG-binding domain protein 2 enhances endothelial angiogenesis and protects mice against hind-limb ischemic injury. Circulation 123, 2964-2974 (2011). doi:10.1161/CIRCULATIONAHA.110.966408 Medline

23. M. Santos, T. Summavielle, A. Teixeira-Castro, A. Silva-Fernandes, S. Duarte-Silva, F. Marques, L. Martins, M. Dierssen, P. Oliveira, N. Sousa, P. Maciel, Monoamine deficits in the brain of methyl-CpG binding protein 2 null mice suggest the involvement of the cerebral cortex in early stages of Rett syndrome. Neuroscience 170, 453-467 (2010). doi:10.1016/j.neuroscience.2010.07.010 Medline

24. N. Chen, M. Zhou, X. Dong, J. Qu, F. Gong, Y. Han, Y. Qiu, J. Wang, Y. Liu, Y. Wei, J. Xia, T. Yu, X. Zhang, L. Zhang, Epidemiological and clinical characteristics of 99 cases of 2019 novel coronavirus pneumonia in Wuhan, China: A descriptive study. Lancet 395, 507-513 (2020). doi:10.1016/S0140-6736(20)30211-7 Medline

25. F. Wang, F. Sun, J. Luo, T. Yue, L. Chen, H. Zhou, J. Zhang, C. Yang, X. Luo, Q. Zhou, H. Zhu, J. Li, P. Yang, F. Xiong, Q. Yu, H. Zhang, W. Zhang, A. Xu, Z. Zhou, Q. Lu, D. L. Eizirik, S. Zhang, C. Y. Wang, Loss of ubiquitin-conjugating enzyme E2 (Ubc9) in macrophages exacerbates multiple low-dose streptozotocin-induced diabetes by attenuating M2 macrophage polarization. Cell Death Dis. 10, 892 (2019). doi:10.1038/s41419-019-2130-z Medline

26. A. J. Covarrubias, H. I. Aksoylar, T. Horng, Control of macrophage metabolism and activation by mTOR and Akt signaling. Semin. Immunol. 27, 286-296 (2015). doi:10.1016/i.smim.2015.08.001 Medline

27. S. J. Bensinger, P. Tontonoz, Integration of metabolism and inflammation by lipidactivated nuclear receptors. Nature 454, 470-477 (2008). doi:10.1038/nature07202 Medline

28. I. U. Agoulnik, M. C. Hodgson, W. A. Bowden, M. M. Ittmann, INPP4B: The new kid on the PI3K block. Oncotarget 2, 321-328 (2011). doi:10.18632/oncotarget.260 Medline

29. L. M. Sly, V. Ho, F. Antignano, J. Ruschmann, M. Hamilton, V. Lam, M. J. Rauh, G. Krystal, The role of SHIP in macrophages. Front. Biosci. 12, 2836-2848 (2007). doi:10.2741/2276 Medline

30. J. Wang, B. J. Wang, J. C. Yang, M. Y. Wang, C. Chen, G. X. Luo, W. F. He, Research advances in the mechanism of pulmonary fibrosis induced by coronavirus disease 2019 and the corresponding therapeutic measures. Zhonghua Shao Shang Za Zhi 36, E006 (2020).

31. Z. Ye, Y. Zhang, Y. Wang, Z. Huang, B. Song, Chest CT manifestations of new coronavirus disease 2019 (COVID-19): A pictorial review. Eur. Radiol. 30, 43814389 (2020). doi:10.1007/s00330-020-06801-0 Medline

32. B. A. Helling, A. N. Gerber, V. Kadiyala, S. K. Sasse, B. S. Pedersen, L. Sparks, Y. Nakano, T. Okamoto, C. M. Evans, I. V. Yang, D. A. Schwartz, Regulation of MUC5B Expression in Idiopathic Pulmonary Fibrosis. Am. J. Respir. Cell Mol. Biol. 57, 9199 (2017). doi:10.1165/rcmb.2017-00460C Medline

33. B. Hu, M. Gharaee-Kermani, Z. Wu, S. H. Phan, Essential role of MeCP2 in the regulation of myofibroblast differentiation during pulmonary fibrosis. Am. J. Pathol. 178, 1500-1508 (2011). doi:10.1016/j.ajpath.2011.01.002 Medline

34. X. Yang, X. Wang, D. Liu, L. Yu, B. Xue, H. Shi, Epigenetic regulation of macrophage polarization by DNA methyltransferase 3b. Mol. Endocrinol. 28, 565-574 (2014). doi:10.1210/me.2013-1293 Medline
35. M. J. Rauh, V. Ho, C. Pereira, A. Sham, L. M. Sly, V. Lam, L. Huxham, A. I. Minchinton, A. Mui, G. Krystal, SHIP represses the generation of alternatively activated macrophages. Immunity 23, 361-374 (2005). doi:10.1016/i.immuni.2005.09.003 Medline

36. E. Sahin, S. Haubenwallner, M. Kuttke, I. Kollmann, A. Halfmann, A. M. Dohnal, L. Chen, P. Cheng, B. Hoesel, E. Einwallner, J. Brunner, J. B. Kral, W. C. Schrottmaier, K. Thell, V. Saferding, S. Blüml, G. Schabbauer, Macrophage PTEN regulates expression and secretion of arginase I modulating innate and adaptive immune responses. J. Immunol. 193, 1717-1727 (2014). doi:10.4049/jimmunol.1302167 Medline

37. J. R. Covvey, E. E. Mancl, Recent evidence for pharmacological treatment of idiopathic pulmonary fibrosis. Ann. Pharmacother. 48, 1611-1619 (2014). doi:10.1177/1060028014551015 Medline

38. M. Gharaee-Kermani, M. R. Gyetko, B. Hu, S. H. Phan, New insights into the pathogenesis and treatment of idiopathic pulmonary fibrosis: A potential role for stem cells in the lung parenchyma and implications for therapy. Pharm. Res. 24. 819-841 (2007). doi:10.1007/s11095-006-9216-x Medline

39. T. C. Carvalho, J. T. McConville, The function and performance of aqueous aerosol devices for inhalation therapy. J. Pharm. Pharmacol. 68, 556-578 (2016). doi:10.1111/iphp.12541 Medline

40. A. Elhissi, Liposomes for Pulmonary Drug Delivery: The Role of Formulation and Inhalation Device Design. Curr. Pharm. Des. 23, 362-372 (2017) doi:10.2174/1381612823666161116114732 Medline

41. V. Ivanova, O. B. Garbuzenko, K. R. Reuhl, D. C. Reimer, V. P. Pozharov, T. Minko, Inhalation treatment of pulmonary fibrosis by liposomal prostaglandin E2. Eur. J. Pharm. Biopharm. 84, 335-344 (2013). doi:10.1016/i.ejpb.2012.11.023 Medline

42. O. B. Garbuzenko, V. Ivanova, V. Kholodovych, D. C. Reimer, K. R. Reuhl, E. Yurkow, D. Adler, T. Minko, Combinatorial treatment of idiopathic pulmonary fibrosis using nanoparticles with prostaglandin E and siRNA(s). Nanomedicine (Lond.) 13, 19831992 (2017). doi:10.1016/i.nano.2017.04.005 Medline

43. K. L. Bajema, A. M. Oster, O. L. McGovern, S. Lindstrom, M. R. Stenger, T. C. Anderson, C. Isenhour, K. R. Clarke, M. E. Evans, V. T. Chu, H. M. Biggs, H. L. Kirking, S. I. Gerber, A. J. Hall, A. M. Fry, S. E. Oliver, G. Abedi, W. Bower, K. Chatham-Stephens, L. Conklin, L. Cooley, M. Cortese, A. Curns, K. Dooling, R. Gokhale, J. Gold, G. Grant, J. Gutman, E. Hesse, S. Kamili, L. Kim, R. Kirkcaldy, E. Koumans, S. Kujawski, G. Langley, J. Lively, X. Lu, B. Lynch, S. Lyss, L. Malapati, M. Martin, S. Mbaeyi, P. McClung, C. Midgley, M. Miller, M. Morales, J. Murray, A. Parker Fiebelkorn, M. Patel, G. Peacock, T. Pierce, B. Rha, S. Sakthivel, E. Schneider, D. A. Siegel, B. Sunshine, M. Wallace, L. Wang, J. Watson, B. Whitaker, A. Yousaf; 2019-nCoV Persons Under Investigation Team; 2019-CoV Persons Under Investigation Team, Persons Evaluated for 2019 Novel Coronavirus - United States, January 2020. MMWR Morb. Mortal. Wkly. Rep. 69, 166-170 (2020). doi:10.15585/mmwr.mm6906e1 Medline

44. J. Kay, K. S. Upchurch, ACR/EULAR 2010 rheumatoid arthritis classification

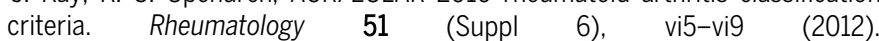
doi:10.1093/rheumatology/kes279 Medline

45. G. Raghu, H. R. Collard, J. J. Egan, F. J. Martinez, J. Behr, K. K. Brown, T. V. Colby, J. F. Cordier, K. R. Flaherty, J. A. Lasky, D. A. Lynch, J. H. Ryu, J. J. Swigris, A. U. Wells, J. Ancochea, D. Bouros, C. Carvalho, U. Costabel, M. Ebina, D. M. Hansell, T. Johkoh, D. S. Kim, T. E. King Jr., Y. Kondoh, J. Myers, N. L. Müller, A. G. Nicholson, L. Richeldi, M. Selman, R. F. Dudden, B. S. Griss, S. L. Protzko, H. J. Schünemann, A. E. J. A. C. I. P. Fibrosis; ATS/ERS/JRS/ALAT Committee on Idiopathic Pulmonary Fibrosis, An official ATS/ERS/JRS/ALAT statement: idiopathic pulmonary fibrosis: evidence-based guidelines for diagnosis and management. Am. J. Respir. Crit. Care Med. 183, 788-824 (2011). doi:10.1164/rccm.2009040GL Medline

46. T. Ashcroft, J. M. Simpson, V. Timbrell, Simple method of estimating severity of pulmonary fibrosis on a numerical scale. J. Clin. Pathol. 41, 467-470 (1988). doi:10.1136/jcp.41.4.467 Medline

47. H. Zhang, P. Zhou, Y. Wei, H. Yue, Y. Wang, M. Hu, S. Zhang, T. Cao, C. Yang, M. Li, G. Guo, X. Chen, Y. Chen, M. Lei, H. Liu, J. Zhao, P. Peng, C. Y. Wang, R. Du, Histopathologic Changes and SARS-CoV-2 Immunostaining in the Lung of a Patient With COVID-19. Ann. Intern. Med. 172, 629-632 (2020). doi:10.7326/M20-0533 Medline

48. Y. C. Guo, M. Zhang, F. X. Wang, G. C. Pei, F. Sun, Y. Zhang, X. He, Y. Wang, J. Song 
F. M. Zhu, N. S. Pandupuspitasari, J. Liu, K. Huang, P. Yang, F. Xiong, S. Zhang, Q. Yu, Y. Yao, C. Y. Wang, Macrophages Regulate Unilateral Ureteral ObstructionInduced Renal Lymphangiogenesis through C-C Motif Chemokine Receptor 2Dependent Phosphatidylinositol 3-Kinase-AKT-Mechanistic Target of Rapamycin Signaling and Hypoxia-Inducible Factor-1 $\alpha /$ Vascular Endothelial Growth Factor-C

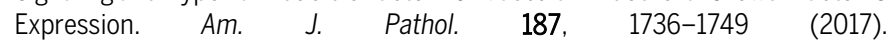
doi:10.1016/i.aipath.2017.04.007 Medline

49. Z. Zhang, L. He, S. Hu, Y. Wang, Q. Lai, P. Yang, Q. Yu, S. Zhang, F. Xiong, S. Simsekyilmaz, Q. Ning, J. Li, D. Zhang, H. Zhang, X. Xiang, Z. Zhou, H. Sun, C. Y. Wang, AAL exacerbates pro-inflammatory response in macrophages by regulating Mincle/Syk/Card9 signaling along with the Nlrp3 inflammasome assembly. Am. J. Transl. Res. 7, 1812-1825 (2015). Medline

50. L. He, F. Sun, Y. Wang, J. Zhu, J. Fang, S. Zhang, Q. Yu, Q. Gong, B. Ren, X. Xiang, Z. Chen, Q. Ning, J. Hu, P. Yang, C. Y. Wang, HMGB1 exacerbates bronchiolitis obliterans syndrome via RAGE/NF- $\mathrm{kB} /$ HPSE signaling to enhance latent TGF- $\beta$ release from ECM. Am. J. Transl. Res. 8, 1971-1984 (2016). Medline

51. H. Chen, X. Xu, J. Teng, S. Cheng, H. Bunjhoo, Y. Cao, J. Liu, J. Xie, C. Wang, Y. Xu, W. Xiong, CXCR4 inhibitor attenuates allergen-induced lung inflammation by down-regulating MMP-9 and ERK1/2. Int. J. Clin. Exp. Pathol. 8, 6700-6707 (2015). Medline

52. X. He, Q. Lai, C. Chen, N. Li, F. Sun, W. Huang, S. Zhang, Q. Yu, P. Yang, F. Xiong, Z. Chen, Q. Gong, B. Ren, J. Weng, D. L. Eizirik, Z. Zhou, C. Y. Wang, Both conditional ablation and overexpression of E2 SUMO-conjugating enzyme (UBC9) in mouse pancreatic beta cells result in impaired beta cell function. Diabetologia 61, 881895 (2018). doi:10.1007/s00125-017-4523-9 Medline

53. P. Yang, Y. Zhang, J. Pang, S. Zhang, Q. Yu, L. He, K. U. Wagner, Z. Zhou, C. Y. Wang, Loss of Jak2 impairs endothelial function by attenuating Raf-1/MEK1/Sp-1 signaling along with altered eNOS activities. Am. J. Pathol. 183, 617-625 (2013). doi:10.1016/i.ajpath.2013.04.007 Medline

54. X. Fu, F. Sun, F. Wang, J. Zhang, B. Zheng, J. Zhong, T. Yue, X. Zheng, J. F. Xu, C. Y. Wang, Aloperine Protects Mice against DSS-Induced Colitis by PP2A-Mediated PI3K/Akt/mTOR Signaling Suppression. Mediators Inflamm. 2017, 5706152 (2017). doi:10.1155/2017/5706152 Medline

55. K. A. Whitehead, J. R. Dorkin, A. J. Vegas, P. H. Chang, O. Veiseh, J. Matthews, O. S. Fenton, Y. Zhang, K. T. Olejnik, V. Yesilyurt, D. Chen, S. Barros, B. Klebanov, T. Novobrantseva, R. Langer, D. G. Anderson, Degradable lipid nanoparticles with predictable in vivo siRNA delivery activity. Nat. Commun. 5, 4277 (2014). doi:10.1038/ncomms5277 Medline

\section{ACKNOWLEDGMENTS}

We are grateful to those patients for donating their BALF and lung tissues for the studies. We are also grateful to Wenye Mo and Siqian Liu for breeding the mice for our studies. Funding: This study was supported by the National Natural Science Foundation of China (81530024, 9174920038, 81920108009, 81770823,81800068 and 81670729), the Ministry of Science and Technology (2016YFC1305002 and 2017YFC1309603), NHC Drug Discovery Program (2017ZX09304022-07), the Department of Science and Technology of Hubei State (2017ACA096), the Integrated Innovative Team for Major Human Disease Programs of Tongji Medical College, Huazhong University of Science and Technology. Author contributions: Y.W. and L.Z was responsible for conducting all experiments and data analyses and wrote the manuscript. G.R.W., H.H.Y., L.Z.R. and T.Y. were involved in Western blot, histological analysis, immunostaining and animal breeding. Q.Z is responsible for liposome preparation. F.X.W. and L.M.C. provided help for flow cytometry. F.S. and J.S. jointly performed some of the experiments. B.W.M., S.Z., F.X., Q.L.Y., P.Y., Y.J.X. and J.P.Z. were involved in study design and review of the manuscript. H.L.Z. contributed to discussion and review of the manuscript, study design, and manuscript preparation. W.N.X. and C.-Y.W. contributed to the study design and manuscript preparation. All authors were involved in drafting the article or revising it critically, and all authors gave their approval for the final manuscript to be published. Competing interests: The authors declare no competing interests. Data and Materials availability: All data needed to evaluate the conclusions in the paper are present in the paper and/or the Supplementary Materials. The data that support the findings of this study are available from the corresponding author upon reasonable request.

\section{SUPPLEMENTARY MATERIALS}

advances.sciencemag.org/cgi/content/full/sciadv.abb6075/DC1

Submitted 6 March 2020

Accepted 29 October 2020

Published First Release 4 December 2020

10.1126/sciadv.abb6075 

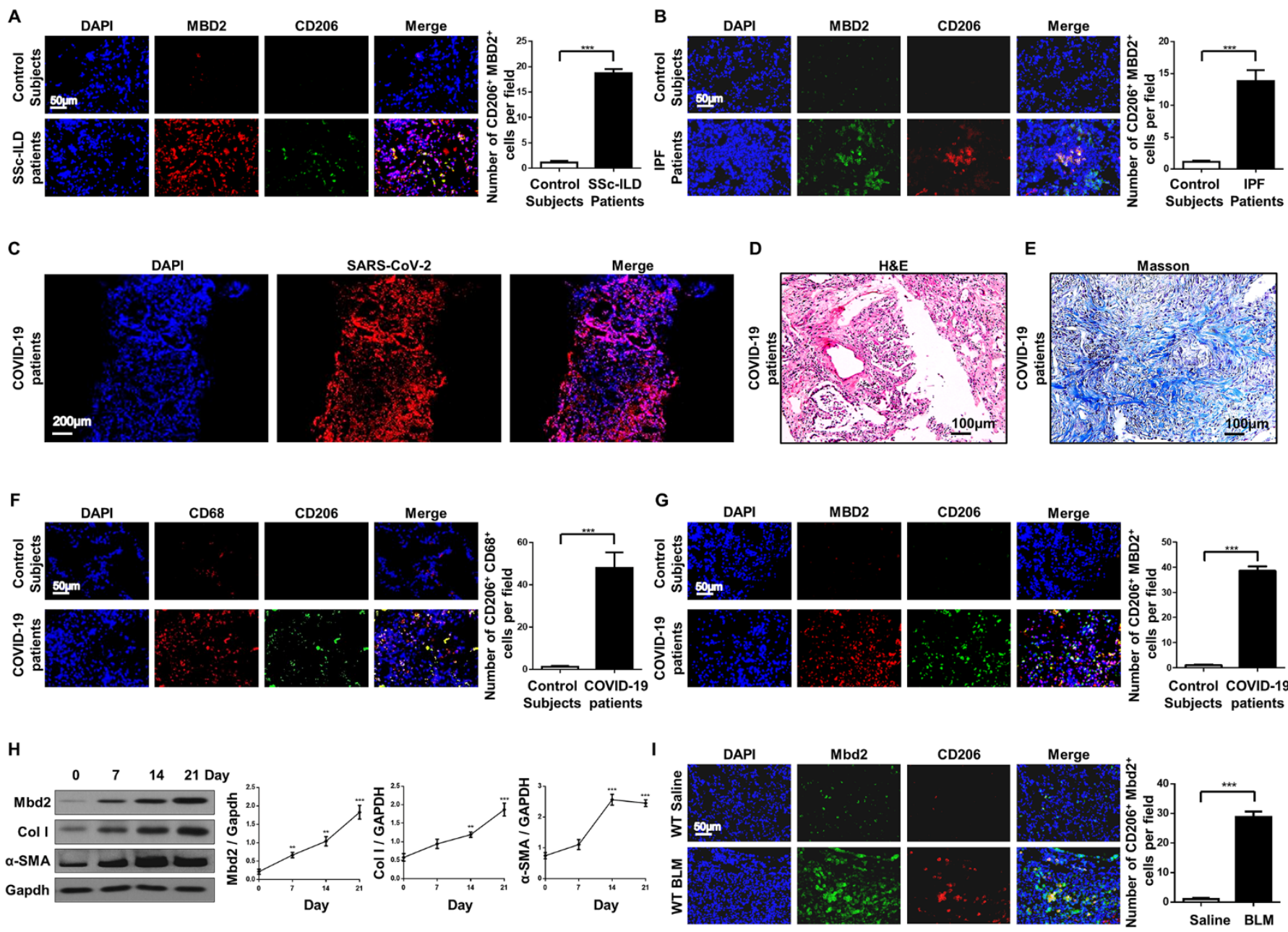

Fig. 1. Analysis of MBD2 expression in PF patients and mice with BLM induction. (A and $B$ ) Representative results for coimmunostaining of MBD2 and CD206 in the lung sections from patients with SSc-ILD (A) and IPF (B). (C) Representative results for immunostaining of SARS-CoV-2 in the lung sections from patients with COVID19. The images were taken under original magnification $\times 100$. (D) Histological analysis $(H \& E)$ of the lung sections from COVID-19 patients. The images were taken under original magnification $\times 200$. (E) Histological analysis (Masson) of the lung sections from COVID-19 patients. The images were taken under original magnification $\times 200$. (F) Representative results for coimmunostaining of CD68 (a macrophage marker) and CD206 (an M2 marker) in the lung sections from COVID-19 patients. (G) Representative results for coimmunostaining of MBD2 and CD206 in the lung sections from COVID-19 patients. $(H)$ Western blot analysis of Mbd2 and Collagen I and $\alpha-S M A$ expression in the lungs of mice following BLM induction. (I) Results for coimmunostaining of Mbd2 and CD206 in BLM-induced lung sections. The nuclei were stained blue by DAPI, and the images were taken under original magnification $\times 400$. A total of two patients with COVID-19, three patients with SSc-ILD, eight patients with IPF and six control subjects were analyzed. Five mice were analyzed in each group. Col I, collagen I. The data are represented as the mean $\pm \mathrm{SD} .{ }^{* *}, p<0.01 ;{ }^{* * *}, p<0.001$. 
A

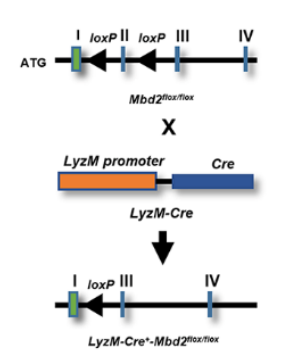

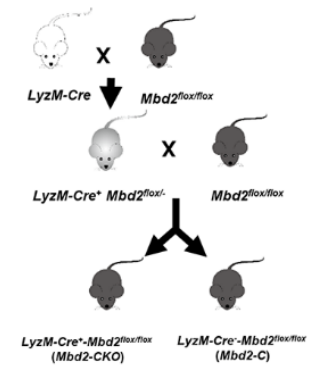

B
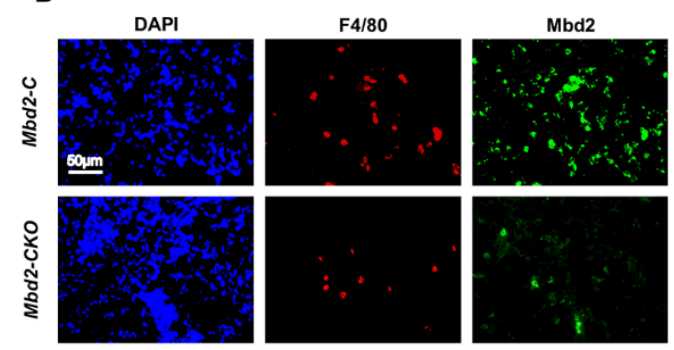

C

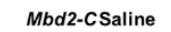

Mbd2-CBLM

Mbd2-CKOSaline

Mbd2-СKOBLM
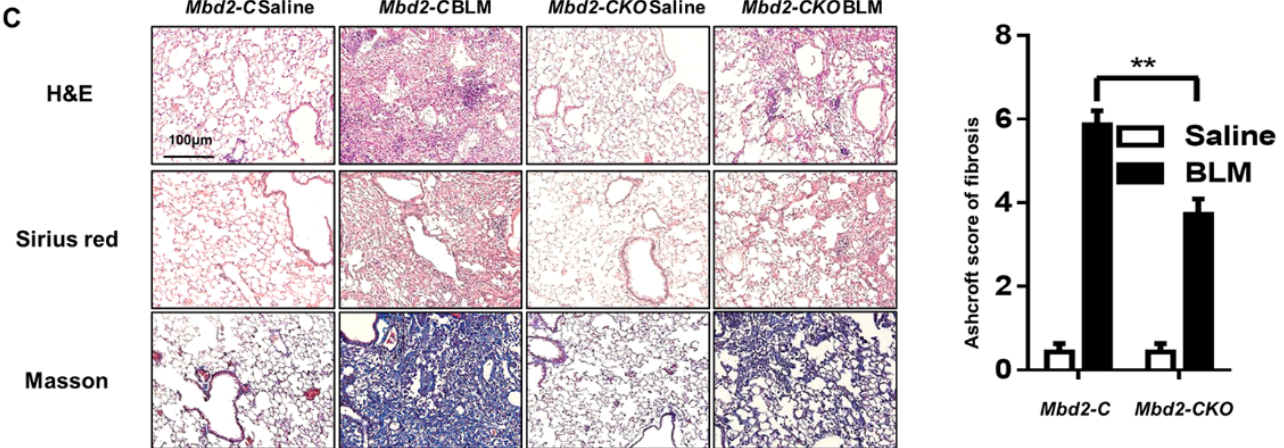

Mbd2-C Mbd2-CKO

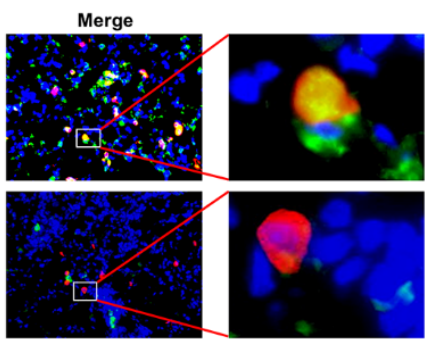

D

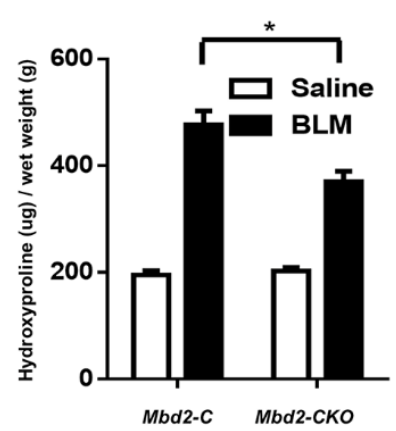

E
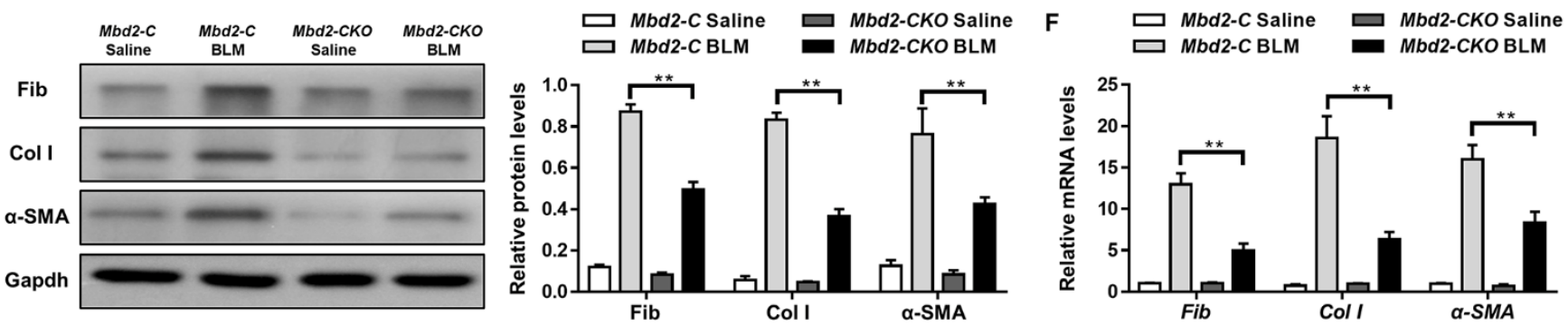

Fig. 2. Comparison of the severity of lung fibrosis between Mbd2-C and Mbd2-CKO mice after BLM induction. (A) Mbd2 flox/flox mice were generated by inserting two loxP sequences in the same direction into the introns flanked with the exon 2 of MBD2 based on the CRISPR-Cas9 system, which could produce a nonfunctional MBD2 protein by generating a stop codon in exon 3 after Cre-mediated gene deletion. Mbd2flox/flox then crossed with the $L y z M-C r e$ transgenic mice to get the macrophage-specific Mbd2-knockout mice which

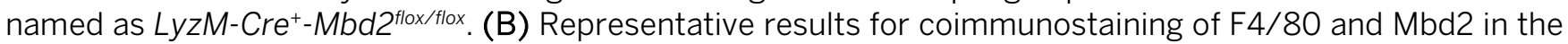
lung sections from Mbd2-C and Mbd2-CKO mice. Nuclei were stained blue by DAPI, and the images were taken at an original magnification of $\times 400$. (C) Histological analysis of the severity of lung fibrosis in mice after BLM induction. Left panel: representative images for H\&E (top), Sirius red (middle) and Masson staining (bottom). Right panel: a bar graph figure showing the quantitative mean score of the severity of fibrosis. Images were captured at $\times 200$ magnification. (D) Quantification of hydroxyproline contents in Mbd2-C and Mbd2-CKO mice after BLM challenge. (E) Western blot analysis of fibronectin, collagen I and $\alpha$-SMA. (F) RT-PCR analysis of fibronectin, collagen I and $\alpha$-SMA. Seven mice were included in each study group. BLM, bleomycin; Col I, collagen I; Fib, fibronectin. The data are represented as the mean $\pm \mathrm{SD}$. ${ }^{*}, p<0.05 ;{ }^{* *}, p<0.01$. 
A
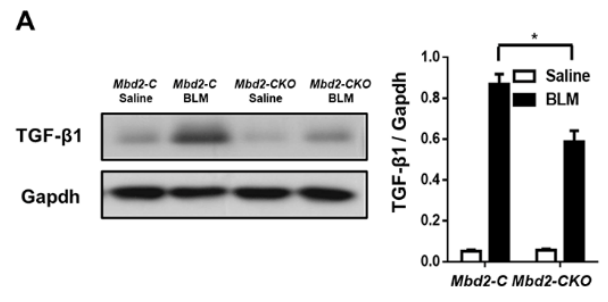

B

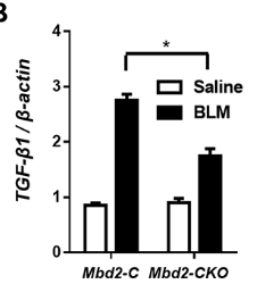

C
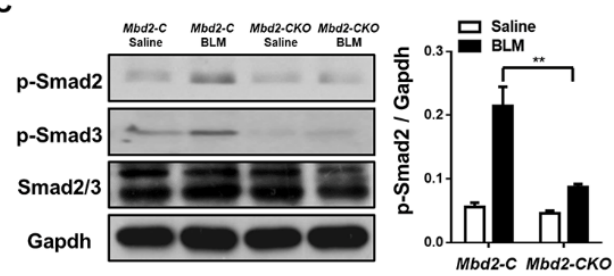

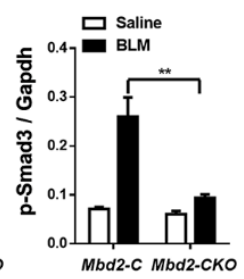

D

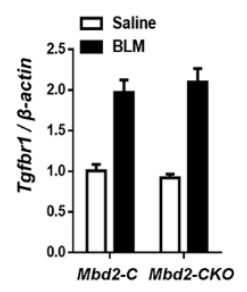

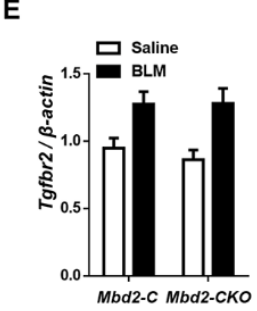

F
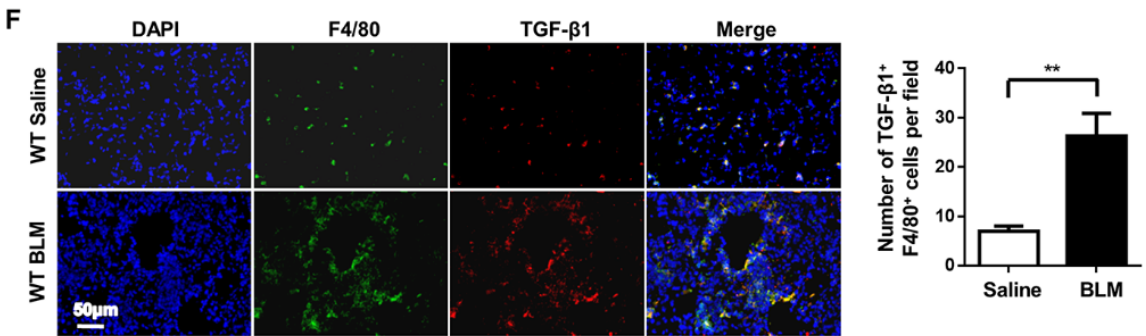

Fig. 3. Mbd2 deficiency repressed TGF- $\beta /$ Smad signaling after BLM induction. (A) Western blot analysis of TGF- $\beta 1$ expression in the lung homogenates. (B) Real-time PCR results for TGF- $\beta 1$ expression in the lungs after BLM induction. (C) Western blot analysis of p-Smad2, p-Smad3 and Smad2/3 expression. Real-time PCR results for Tgfr1 (D) and Tgfr2 (E) expression in the lungs after BLM induction. (F) Coimmunostaining of TGF- $\beta 1$ and F4/80 in the lung sections of WT mice. The images were taken under original magnification $\times 400$. BLM, bleomycin. Each bar represents the mean \pm SD of seven mice studied. * $p<0.05 ;{ }^{* *}, p<0.01$. 
A

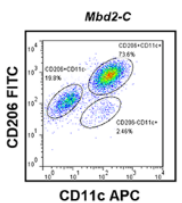

D

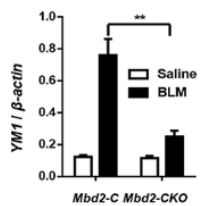

G

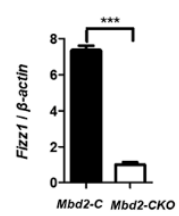

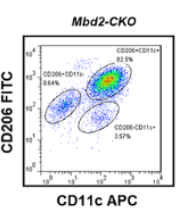

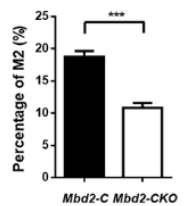

E
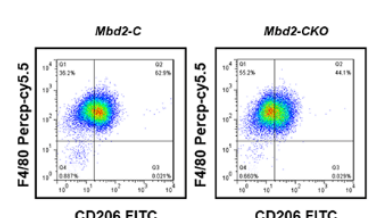

CD206 FITC

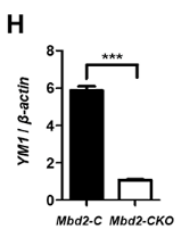

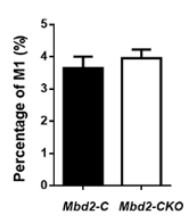

I

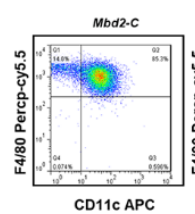

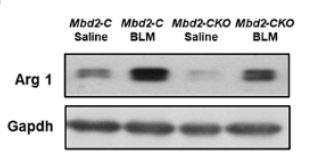

F
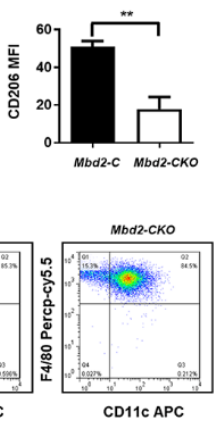

J
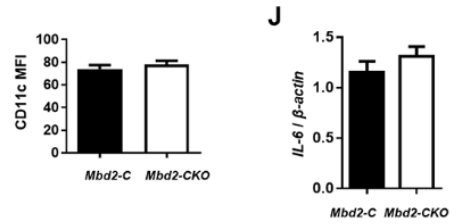

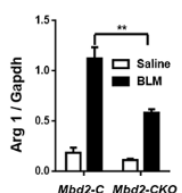

C
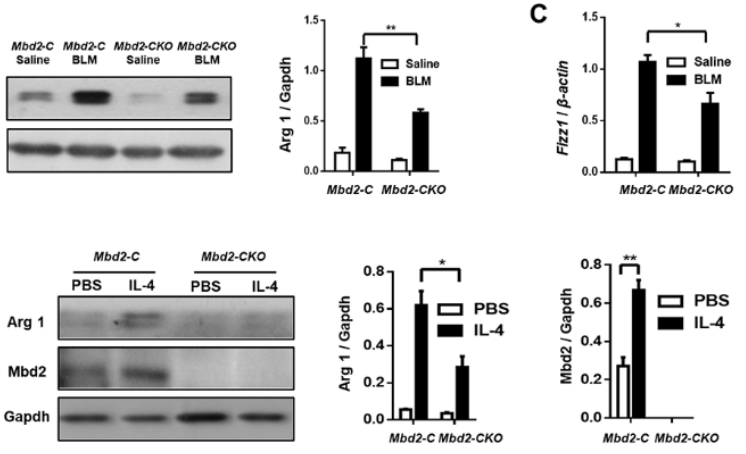

K

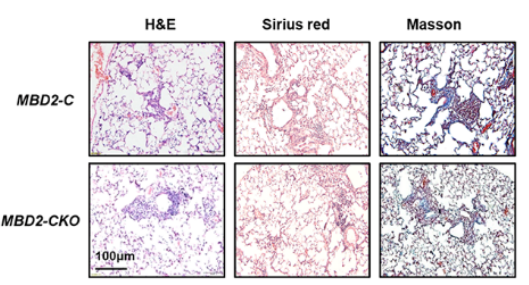

M

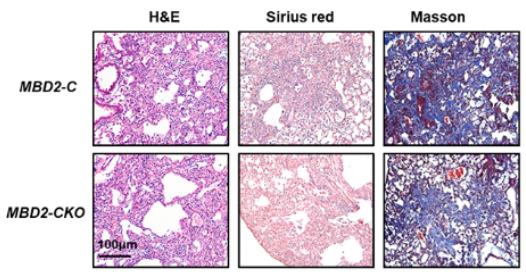

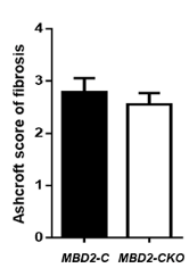

L
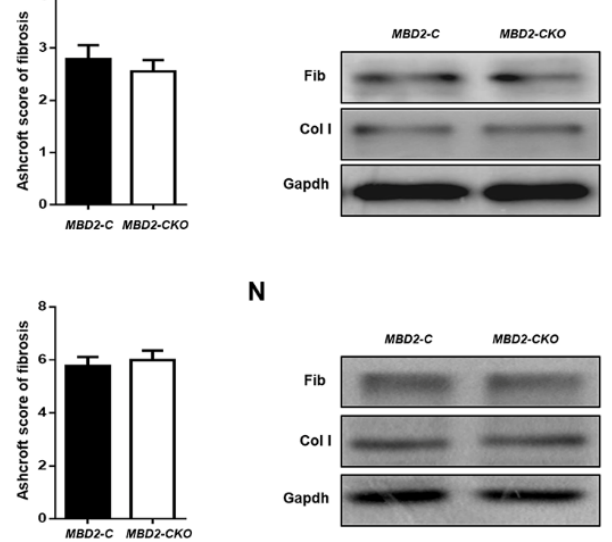

$\mathbf{N}$

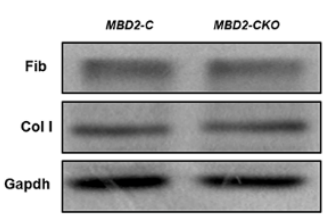

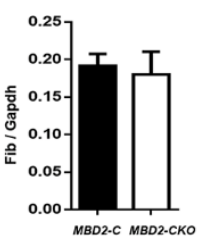
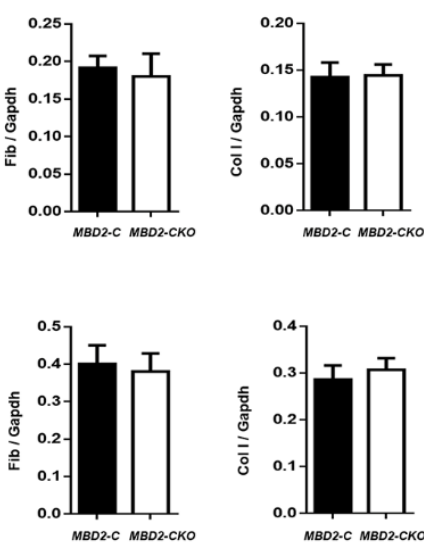

Fig. 4. Mbd2 was overexpressed by the infiltrated M2 macrophages in the lung following BLM induction. (A) Flow cytometry analysis of macrophages derived from lung tissues of $\mathrm{Mbd2}-\mathrm{C}$ and $\mathrm{Mbd2}-\mathrm{CKO}$ mice after BLM induction. (B) Results for Arg 1 expression in the lung homogenates. Real-time PCR results for analysis of Fizz1 (C) and YM1 (D) expression in the lung. (E) Flow cytometry analysis of CD206 expression in BMDMs following IL-4 stimulation. (F) Results for Arg 1 and Mbd2 expression in the BMDMs after IL-4 induction. Real-time PCR for analysis of Fizzl $(\mathrm{G})$ and $Y M 1(\mathrm{H})$ expression in the BMDMs after IL-4 induction. (I) Flow cytometry analysis of CD11c expression in BMDMs. (J) Real-time PCR analysis of IL-6 expression. (K) The severity of pulmonary fibrosis in Mbd2-C and Mbd2-CKO mice after depletion of macrophages. Left panel: representative results for HE, Sirius red and Masson staining. Right panel: a bar graph figure showing the semiquantitative Ashcroft scores for the severity of fibrosis. (L) Levels of fibronectin and collagen I in the lungs of macrophage-depleted Mbd2-C and Mbd2-CKO mice after BLM induction. (M) Results for adoptive transfer of WT macrophages into Mbd2-C and Mbd2-CKO mice following BLM induction. Left panel: representative results for H\&E, Sirius red and Masson staining. Right panel: the semiquantitative Ashcroft scores relevant to the severity of fibrosis. (N) Western blotting for analysis of collagen I and fibronectin expression. All images were captured at $\times 200$ magnification, and seven mice were included in each study group. BLM, bleomycin; Arg 1, arginase 1; BMDMs, bone marrow-derived macrophages; Fizz1, found in inflammatory zone 1; YM1, chitinase 3-like 3; Col I, collagen I; Fib, fibronectin; MFI, mean fluorescence intensity. The data are represented as the mean \pm SD. ${ }^{*}, p<0.05 ;{ }^{* *}, p<0.01$; 

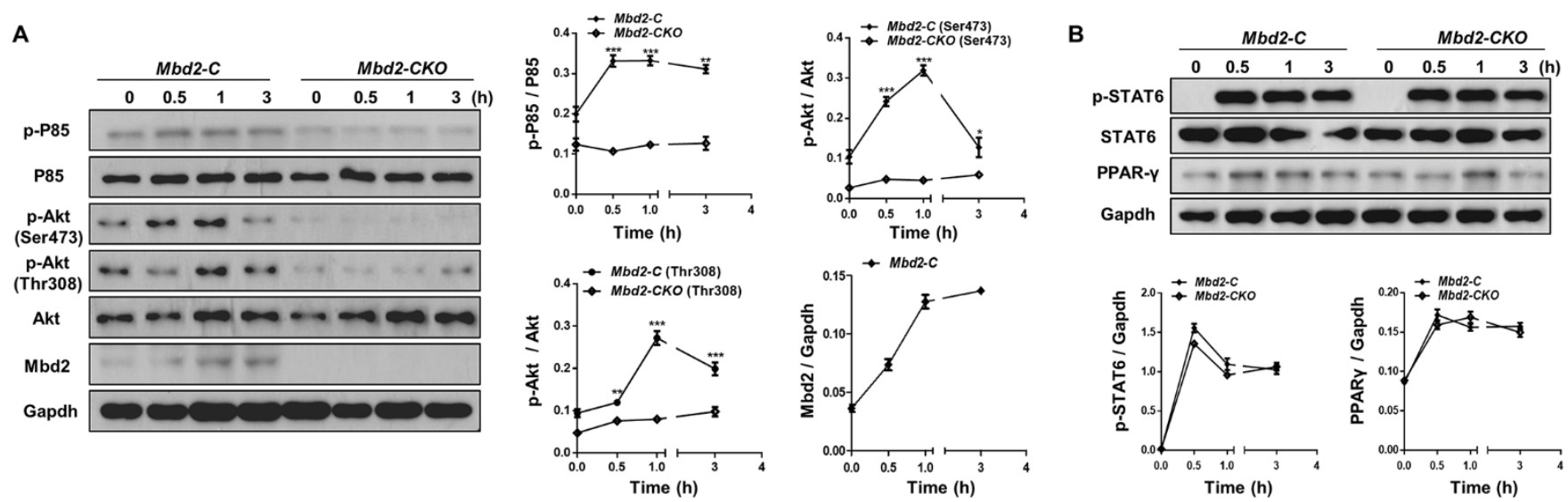

C

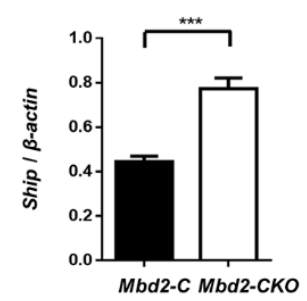

D

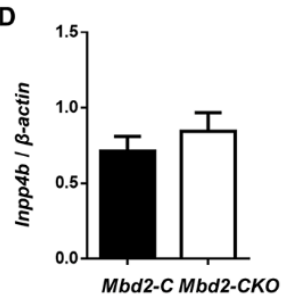

E

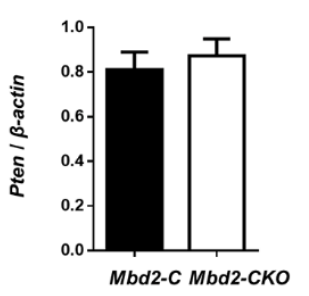

F
G

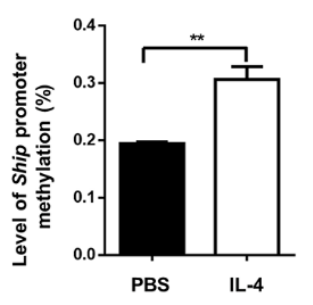

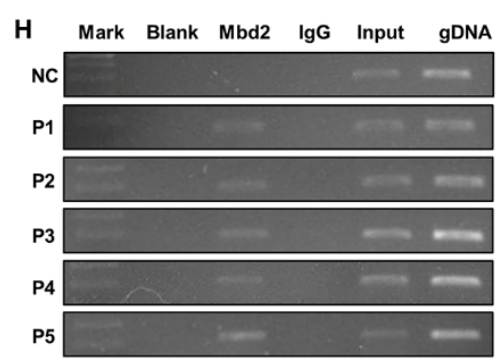

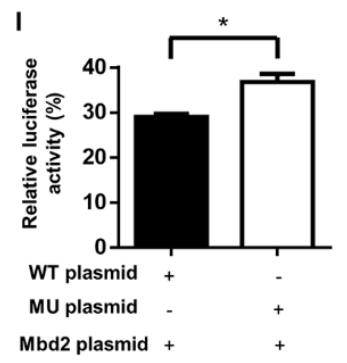

$\mathbf{J}$
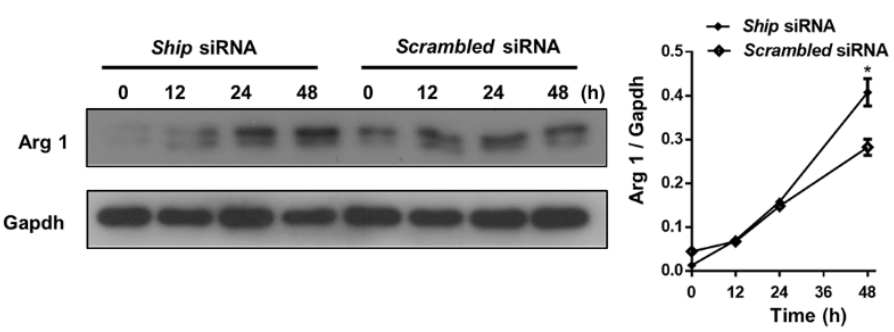

Fig. 5. Loss of Mbd2 attenuated IL-4-induced PI3K/Akt signaling in macrophages. (A) Analysis of IL-4induced PI3K/Akt signaling in macrophages. (B) Results for time-course Western blot analysis of STAT6, p-STAT6 and PPAR-y expression in BMDM following IL-4 stimulation. Real-time PCR for analysis of Ship (C), Inpp4b (D) and Pten (E) expression in IL4-induced BMDMs. (F) Global DNA methylation rate in BMDMs after IL-4 treatment. (G) Results for the bisulfite DNA sequencing analysis of the Ship promoter. (H) ChIP results for the analysis of Mbd2 binding activity to the Ship promoter. The distal region of Ship promoter ($1770 b p$ to $-1540 b p$ ) was served as a negative control. (I) Relative luciferase activity in BMDMs. (J) Results for a time-course Western blot analysis of Arg 1 expression in IL-4-induced Mbd2-CKO BMDMs transfected with Scrambled or Ship siRNA. BMDMs, bone marrow-derived macrophages; Ship, SH2-containing inositol 5'-phosphatase; Inpp4B, nositolpolyphosphate 4-pho sphatase type II; Pten, phosphatase and tensin homolog; Arg 1, arginase 1; NC, negative control. The data are represented as the mean \pm SEM. * $p<0.05$; $*^{*}, p<0.01 ;{ }^{* *}, p<0.001$. 

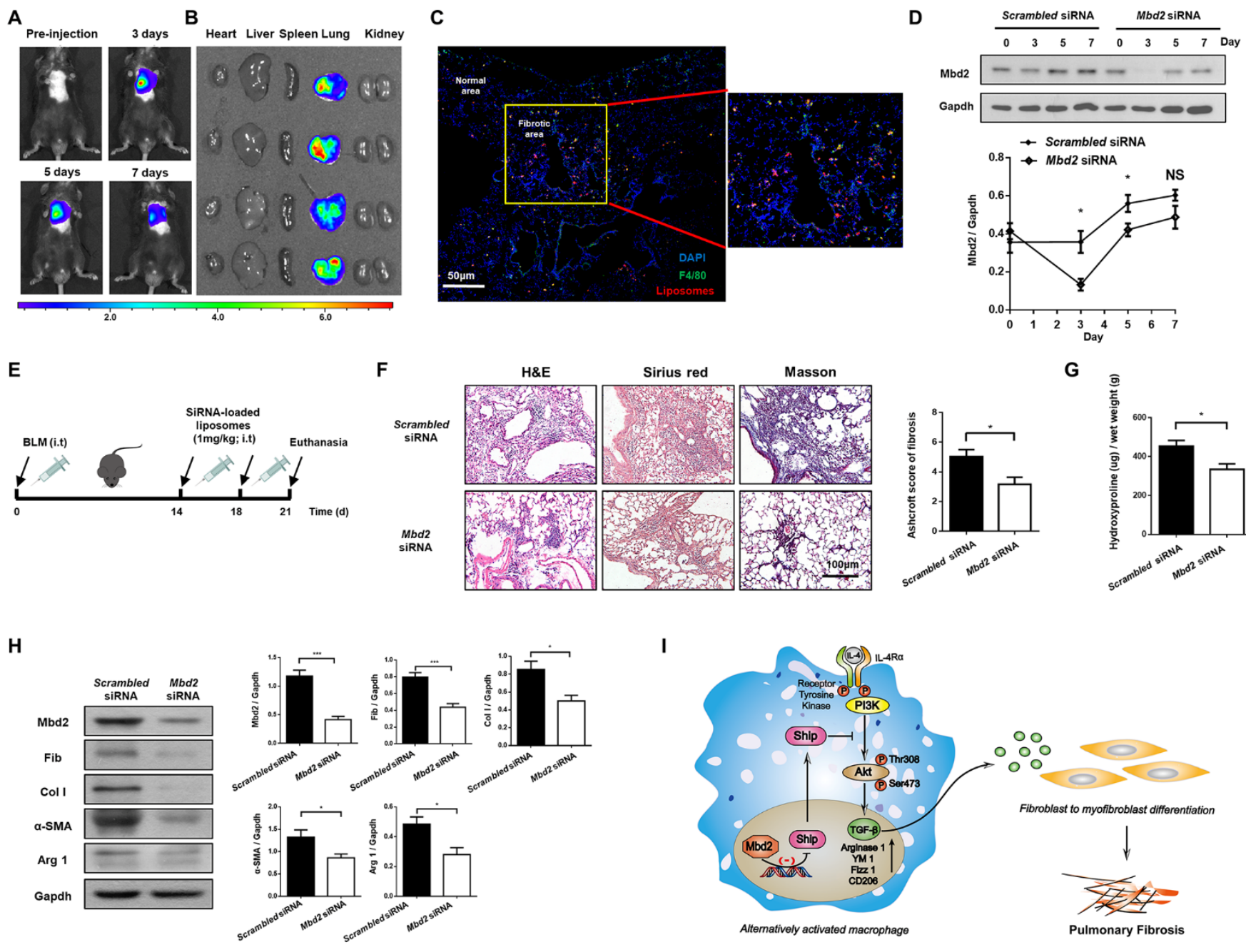

H

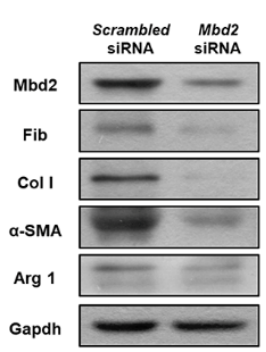

\begin{abstract}
$\mathbf{F}$
\end{abstract}
G

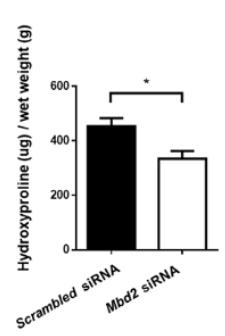

Fig. 6. Intratracheal administration of Mbd2 siRNA-loaded liposomes protected mice from BLM-induced lung injury and fibrosis. (A) Representative IVIS images of the mouse administrated with DiR-labeled liposomes. (B) Ex vivo fluorescence images of major organs from mice. (C) Confocal immunofluorescence image for the biodistribution of liposomes in lungs from BLM-induced mice. Images were captured at $\times 200$ magnification. (D) Temporal Mbd2 expression changes in the lungs from liposome administered mice, and four mice were included in each study group. (E) Schematic for experimental design and timeline of bleomycin-treated WT mice administered with either Scrambled or Mbd2 siRNA-loaded liposomes. (F) Intratracheal administration of Mbd2 siRNA-loaded liposomes provided protection for mice against BLMinduced lung injury and fibrosis. Left panel: representative results for H\&E, Sirius red and Masson staining, right panel: the semiquantitative Ashcroft scores relevant to the severity of fibrosis. Images were captured at $\times 200$ magnification. (G) Quantification of hydroxyproline contents in mice after BLM challenge. Six mice were included in each study group. (H) Western blot analysis of Mbd2, fibronectin, collagen I, $\alpha$-SMA and Arg 1 expression in the lungs. (I) Mbd2 selectively bound to the Ship promoter in macrophages, by which it repressed Ship expression and enhanced PI3K/Akt signaling to promote macrophage M2 program. Upon activation, M2 macrophages secreted high levels of TGF- $\beta 1$ into the milieu of lung fibroblasts, which then induced the progression of pulmonary fibrosis. BLM, bleomycin; i.t, intratracheal injection; Col I, collagen I; Fib, fibronectin; Arg 1, arginase 1. The data are represented as the mean $\pm \mathrm{SD}$. ${ }^{*}, p<0.05 ;{ }^{* *}, p<0.001$. 